\title{
Article \\ Genotype-dependent variation of nutritional quality-related traits in quinoa seeds
}

\author{
Sara Granado-Rodríguez¹, Susana Vilariño-Rodríguez², Isaac Maestro-Gaintán¹, Javier Matías³, María José \\ Rodríguez ${ }^{4}$, Patricia Calvo ${ }^{4}$, Verónica Cruz ${ }^{3}$, Luis Bolaños ${ }^{1}$, María Reguera $^{*}$
}

1 Departamento de Biología, Universidad Autónoma de Madrid, c/Darwin 2, Campus de Cantoblanco, 28049,

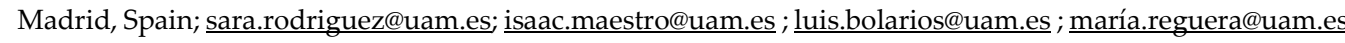
2 Vitrosur Lab SLU. Algodonera del Sur, Carretera Trebujena C-441 (km 5.5), Lebrija, 41740, Sevilla, Spain; svilarino@algosur.com

3 Agrarian Research Institute "La Orden-Valdesequera" of Extremadura (CICYTEX). 06187, Badajoz, Spain javier.matias@juntaex.es ; veronica.cruz@juntaex.es

4 Technological Institute of Food and Agriculture of Extremadura (CICYTEX). 06007, Badajoz, Spain. mariajose.rodriguezg@juntaex.es; patricia.calvo@juntaex.es;

* Correspondence: María Reguera maria.reguera@uam.es ; Tel.: +34 914978189

\begin{abstract}
Exploiting the relationship between the nutritional properties of seeds and the genetic background, constitutes an essential analysis which contributes to broadening our knowledge regarding the control of the nutritional quality of seeds or any other edible plant structure. This constitutes an important aspect when aiming at improving the nutritional characteristics properties of crops, including those of Chenopodium quinoa Willd (quinoa) which is intended to be one of the main nutrient sources ensuring food security worldwide. Changes in the nutritional properties of quinoa seeds due to the influence exerted by the environment, the genotype, or their interaction, have been already described in previous works, but there is an important limitation in the analyses carried out, including the outcomes that can be translated into agronomical practices by which quality can be improved selecting the most adequate genotype. In the present study, several seed nutritional-related parameters from fifteen quinoa cultivars grown in a particular environmental context were analyzed aiming at targeting compounds that can be determinants of seed quality. Important agronomical and nutritional differences were found among cultivars such as distinct mineral or protein contents and seed viability. More importantly, our analyses revealed key correlations between seed quality-related traits in some cultivars, including those that relate yield and antioxidants or the germination rate. These results highlight the importance of considering the genotypic variation in quinoa when selecting improved quinoa varieties with the best nutritional characteristics for new cultivation environments.
\end{abstract}

Keywords: quinoa; genotype; nutritional traits; seed quality;

\section{Introduction}

Quinoa (Chenopodium quinoa Willd.) is a halophytic crop that belongs to the Amaranthaceae family. It can be adapted to a wide variety of agroecosystems and is resistant to stressful environmental conditions [1], [2], possessing as well excellent nutritional properties [3], [1]. All these characteristics have resulted in a global expansion of quinoa cultivation during the past three decades [4] and, consequently, this explains why quinoa can be found from the Andean Altiplano, with harsh climatic conditions associated with high altitudes (reaching over $3500 \mathrm{~m}$ above sea level), to coastal areas.

Quinoa was first domesticated by pre-Columbian cultures more than 7000 years ago, being one of their main sources of nutrients as a good substitute for the lack of animal protein. After the Spanish conquest, quinoa was highly rejected but maintained by indigenous farmers despite the introduction of Old-World species. These farmers domesticated 
the cultivars preserving the genetic diversity found currently in quinoa [5]. This genetic diversity can be divided into different ecotypes that include thousands of accessions $(16,422)[6]$ that reflect the diffusion from the center of origin around Lake Titicaca [5].

Currently, quinoa is still the principal protein source in many areas of the Altiplano. The nutritional value of quinoa seeds was rediscovered during the last decades of the 20th century, leading to a renewal of its production [7]. This led to a boost of spreading its cultivation from very few countries growing this crop in the 80s to 123 countries in 2018 [4]. The success in its international acceptance has been possible mainly due to the nutritional characteristics of the seeds. They resemble cereal seeds due to the high starch content and overall morphology, which explains why quinoa is considered a pseudocereal. Quinoa seeds are gluten-free, have a low glycemic index, being low in sugar and calories but containing an excellent balance of essential amino acids, and high contents of fiber, lipids, carbohydrates, minerals, and bioactive compounds such as vitamins (B2 and E), carotene, tocopherols, and other molecules with antioxidant properties like flavonoids and other phenolic compounds [8-12]. Antioxidants are of economic interest since they can minimize the rancidity and increase the shelf-life of food products [13], but they are also of nutritional interest due to their health-related benefits. Antioxidants have been found to reduce the risks of cancer and cardiovascular disease, and to present anti-inflammatory and anti-microbial activity [14,15]. Quinoa seeds are a good source of antioxidants, exceeding the antioxidant capacity of cereals [16].

Furthermore, quinoa also shows a unique fiber, lipid (with a high ratio of omega-6: omega-3), micro- and macronutrient profiles (often higher than cereal-based products) that give quinoa seeds beneficial characteristics such as decreasing the risk of cancer, cardiovascular and inflammatory diseases, decreasing blood pressure, diabetes, development of hemorrhoids and weight control [17], improving intestinal health [9]. Due to all these characteristics, providing not only nutritional services but health benefits, quinoa is considered a "superfood of the future".

Furthermore, quinoa is offered as a nutritious food for low-income countries and constitutes a crop able to grow on marginal lands (including those with limited rainfalls or poor soil quality) which are not suitable for other major crops [8]. This brings interesting opportunities for the agriculture of low-income countries and, generally, for those countries where agricultural water supply is or will be soon limited. These include Mediterranean countries where there is an urgent need to develop sustainable practices to mitigate the impacts of climate change and human pressure on soil resources [8] which is especially relevant within the current climate change and food security context [18]. Besides, it should be noted that quinoa is not only consumed by humans, as its different plant parts can be used as a nutritionally valuable forage crop, apt for feeding sheep, pigs, cattle, poultry, and horses [19].

Importantly, it should be noted that quinoa exhibits a strong variability of cultivarspecific responses to environmental variation different environmental conditions have been described in quinoa for some seed quality-related parameters including seed size, protein or mineral contents depending on the specific genotype [20-24]. Thus, different cultivars of quinoa have shown substantial differences in the nutritional characteristics which also vary with the environment. What is still unclear is if the parameters that were evaluated are stable among cultivars at different locations or if steady correlations can be found between nutritional-related parameters. A recent work by Granado-Rodriguez et al. [25] showed that some quinoa cultivars, Titicaca and Vikinga, present better qualityrelated traits (including higher protein contents) despite not being the most productive when growing in the Northwestern part of Spain. In line with this, it is key to better understand the genetic and environmental factors determining the nutritional characteristics of quinoa selecting the best adapted genotypes for a particular cultivation environment in 
terms of yield potential, biotic and abiotic stress tolerance but also considering the different nutritional traits. This will be achieved through the use of conventional and molecular tools that will help to unlock the rich biodiversity and cultivation potential of this crop [26].

Therefore, aiming at contributing to unravel genetic differences associated with different genotypes, in this study we analyzed a variety of nutritional-related parameters in fifteen different quinoa varieties with cultivation potential in the Southern region of Spain. Differences in the parameters analyzed were found among varieties for most of the parameters analyzed including plant height, panicle length, mildew incidence, lodging, seed weight and area, protein content, germination rates and seed viability, mineral contents (except for Mg), antioxidants, and saponin contents. Also, important correlations between different seed quality-related parameters (including yield and antioxidants or germination rates) which are genotype-dependent support the presence of genetic determinants of nutritional quality in quinoa.

\section{Results}

\subsection{Plant performance and physiological traits}

In this study, seeds of fifteen different quinoa cultivars were sown on January 27th of 2018 and plants were harvested on either July 18th or August 1st aiming to analyze different nutritional traits under filed conditions (Supplementary Figure 1). Cultivars 'ASE-03', 'A-SE-06', 'A-SE-07', 'A-SE-09', 'A-SE-12', 'A-SE-13', and 'A-SE-15' were harvested in July (Harvesting 1, Supplementary Figure 1), while cultivars 'A-SE-01', 'A-SE-02', 'A-SE04', 'A-SE-05', 'A-SE-08', 'A-SE-10', 'A-SE-11', and 'A-SE-14' presented longer life cycles, and were harvested later (Harvesting 2, Supplementary Figure 1). Total seed yield varied among cultivars, being A-SE- $08 \mathrm{cv}$. the cultivar that presented the highest seed yield (4.7 $\mathrm{t} / \mathrm{ha}$ ) followed by A-SE-11 cv. and A-SE-05 cv. (with 3.5 and $3.4 \mathrm{t} / \mathrm{ha}$, respectively), and ASE-01 the cultivar with the lowest seed yield (0.96 t/ha). Precipitations along the growing season were concentrated in the first months of cultivation (February to April) coinciding with plant nascence and emergence (Supplementary Figure 1) while scarce precipitations were registered from flowering to harvesting time (May-July). Daily mean temperature varied from $8.7^{\circ} \mathrm{C}$ (at sowing) to $25.7^{\circ} \mathrm{C}$ and $30.7^{\circ} \mathrm{C}$ (at harvesting of short- and long-lifecycle cultivars, respectively) increasing progressively along the growing season (Supplementary Figure 1). Inflorescences started appearing in May. Temperatures higher than $25^{\circ} \mathrm{C}$ were registered (for all cultivars) at seed maturation stage.

Plant height also showed significant variations among cultivars (Figure 1). At early stages (82 d.a.s) A-SE-05 cv., A-SE-03 cv. and A-SE-06 cv. were the tallest, with $54 \pm 5.76 \mathrm{~cm}$ of plant length while at middle stages (100 d.a.s.), A-SE-03 cv. and A-SE-06 cv. were the highest with $130.4+8.94 \mathrm{~cm}$ and $130.4 \pm 6.33 \mathrm{~cm}$ height, respectively. At latest stages (128 d.a.s), A-SE-07 cv. and A-SE- $15 \mathrm{cv}$. were the tallest plants presenting $170.90 \pm 22.87 \mathrm{~cm}$ and $163.40 \pm 31.99 \mathrm{~cm}$ height, respectively. On the other hand, A-SE-04 cv. was the shortest cultivar throughout the time going from $14.9 \pm 5.27 \mathrm{~cm}$ height at an early stage, to $69.10 \pm$ $11.74 \mathrm{~cm}$ height at middle stage and $117.30 \pm 25.12 \mathrm{~cm}$ at the end of the life cycle. In line with this parameter, lodging was also evaluated in this study. Thus, it was observed that the cultivars A-SE-07 and A-SE-03 presented greater lodging resistance (3\% of lodging plants), while the cultivar A-SE-12 showed great sensitivity to lodging with $36 \%$ of affected plants (Supplementary Figure 2). Interestingly, lodging resistance was not related to panicle weight as the cultivars with contrasting lodging resistance (A-SE-07, A-SE-03, and A-SE-12) did not show significant differences in panicle lengths. 
A

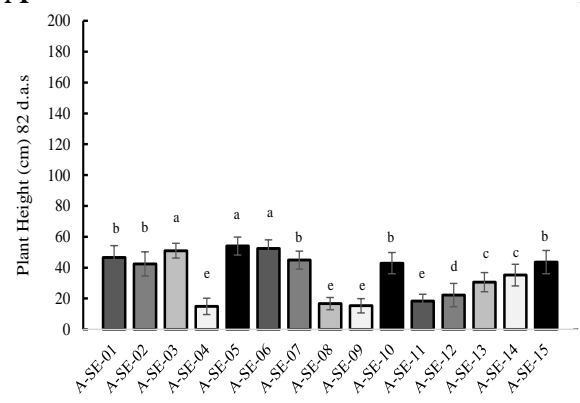

B

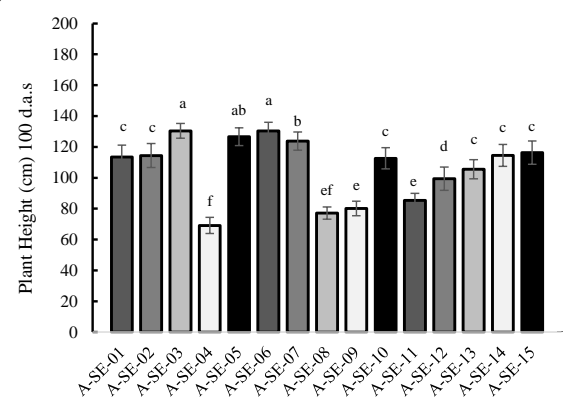

C

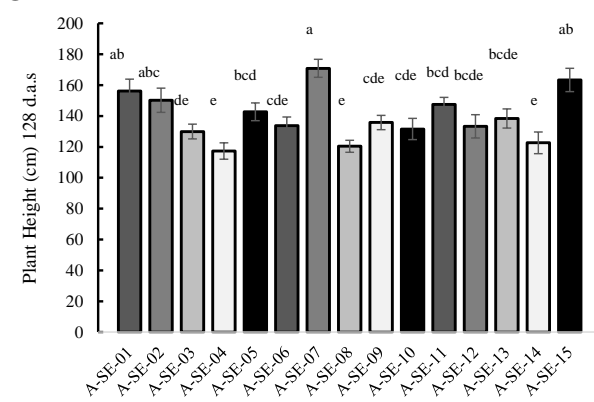

Figure 1. Plant height. Plant height $(\mathrm{cm})$ was determined in the 15 cultivars analyzed at A) 82 , B) 100 and C) 182 d.a.s. Error bars represent the standard deviation. Bars that do not share the same letters show statistically significant differences following the Krustal-Wallis at a $p$-value $<0.05$ for 82 d.a.s. and 100 d.a.s. and ANOVA test and Tukey post-hoc test at a $p$-value $<0.05$ for 128 d.a.s.

Panicle length was determined in the different cultivars evaluated after 128 d.a.s . Most of the cultivars showed panicle lengths between 30 and $40 \mathrm{~cm}$, with A-SE-01 cv. having the lowest values $(21.70 \pm 2.53 \mathrm{~cm})$ and A-SE-13 cv. showing the highest $(40.40 \pm 3.03$ $\mathrm{cm}$ ) (Figure 2). In addition to the panicle length, the weight of those panicles was also measured (Supplementary Figure 2). The results pointed that having larger panicles usually correlated with heavier weights although some exceptions were observed (i.e. A-SE$02 \mathrm{cv}$. was $33.8 \pm 3.67 \mathrm{~cm}$ length but presented the biggest weight, $3.20 \mathrm{~kg} / 25$ panicles).
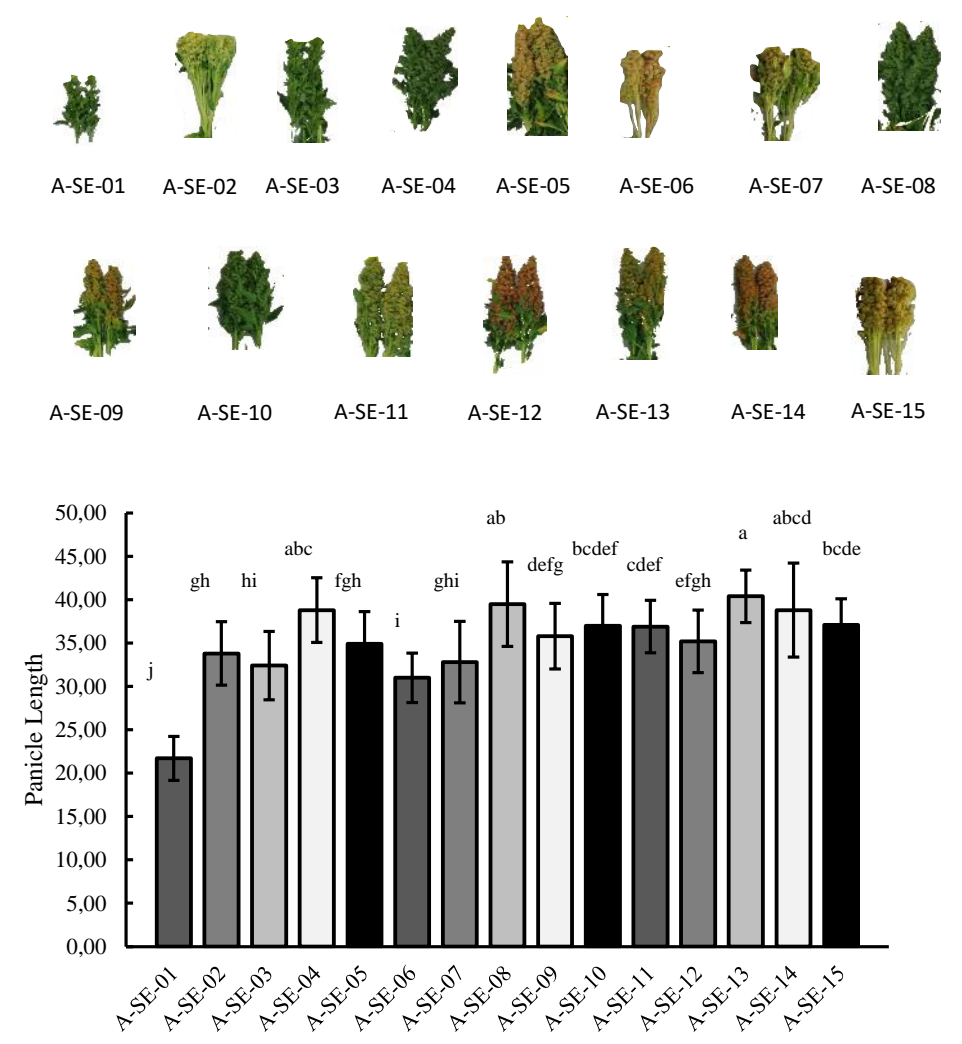

Figure 2. Panicle lenght. Panicle length $(\mathrm{cm})$ was determined in the 15 cultivars analyzed. . Error bars represent the standard deviation. Bars that do not share the same letters show statistically significant differences following the Krustal-Wallis test at a $p$-value $<0.05$ 
Mildew incidence and severity were analyzed throughout the experiment (at 82, 100, and 128 d.a.s.) (Figure 3). A-SE-03 cv. and A-SE-08 cv. were the less affected cultivars at early stages and A-SE-06 cv. and A-SE-12 cv. at later stages, meanwhile A-SE-09 cv., ASE-10 cv., and A-SE-11 cv. were the most stricken ones in terms of severity, especially at 128 d.a.s.

A

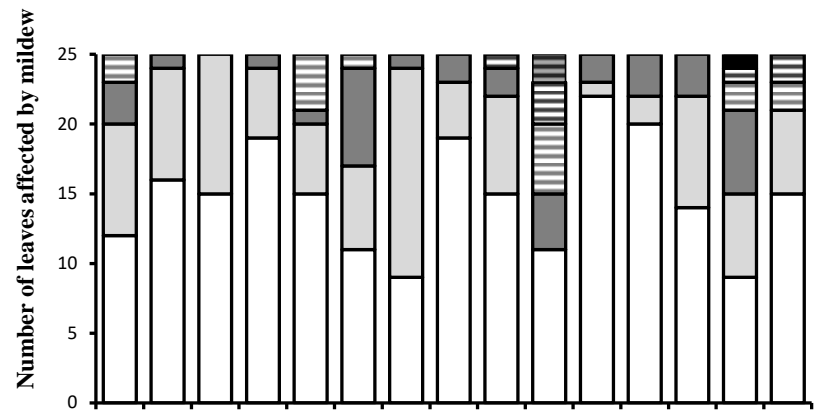

Mildew Severity

B

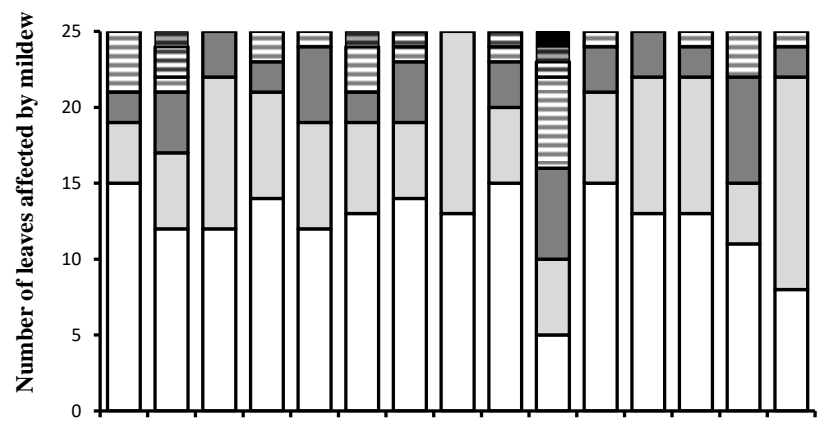

$\square 0 \%$

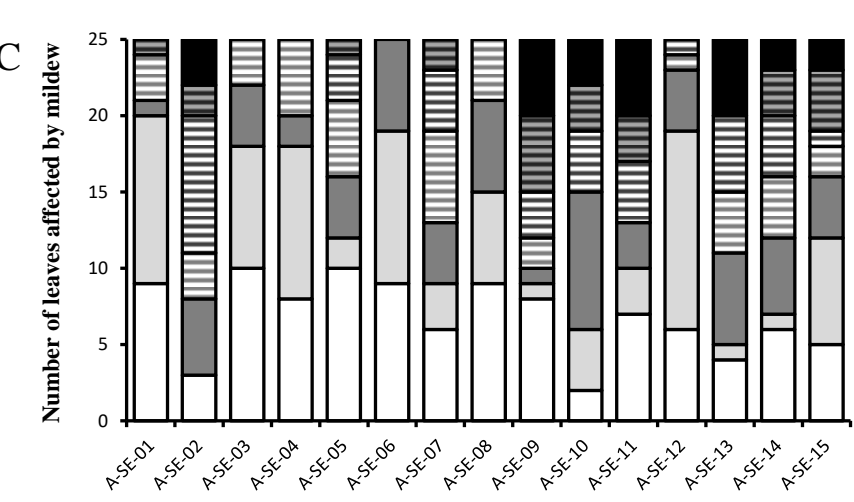

$\square 1-10 \%$

$\square 11-20 \%$

曰 $30 \%$

目 $40 \%$

目 $50 \%$

$60 \%$

Figure 3. Mildew incidence and severity. Mildew incidence and severity were determined as described in the Methods section. Different degree of severity was considered as the percentage (\%) of leaf affected by the pathogen (leaf are converage of $0 \%, 1-10 \%, 11-20 \%, 30,40 \%, 50 \%$, or more than $60 \%$ ). Mildew incidence and severity were evaluated at different developmental stages: at A) 82 (upper panel), B) 100 (middle panel), and C) 128 d.a.s (bottom panel).

Seed weight showed an effect related to the cultivar (Figure 4A). The cultivars A-SE05 cv., A-SE-13 cv. and A-SE-15 cv. presented the heaviest seeds while A-SE-04 cv., A-SE$10 \mathrm{cv}$., and A-SE-09 cv. showed the lightest seed weights. Thus, in order from the heavier to the lighter seed weights, cultivars would be organized as follows: (A-SE-05 cV.=A-SE$13 \mathrm{cv}$. $)>$ A-SE-15 cv. $>($ A-SE-06 cv. $=$ A-SE-11 cv. $)>($ A-SE-02 cv. $=A-S E-12 \mathrm{cv} .=A-S E-14 \mathrm{cv})>.(\mathrm{A}-$ SE-01 cv.=A-SE-07 cv.) $>$ (A-SE-03 cv. =A-SE-08 cv.)>A-SE-09 cv. $>$ (A-SE-04 cv.=A-SE-10 cv.)

Seed area showed a high correlation with seed weight (Supp. Figure3). A-SE-05 cV., A-SE-03 cv., and A-SE-15 cv. presented the largest seeds while A-SE-04 cv., A-SE-08 cv., A-SE-09 cv., and A-SE-10 cv. had the narrowest seed areas (Figure 4B). 
Figure 4. Seed weight and seed area. A) Seed weight $(\mathrm{g})$ and B) area $\left(\mathrm{mm}^{2}\right)$ were determined among the different cultivars studied. Error bars represent the standard deviation. Bars that do not share the same letters show statistically significant differences following a Krustal-Wallis test by ranks for multiple comparisons at a $p$-value $<0.05$

A

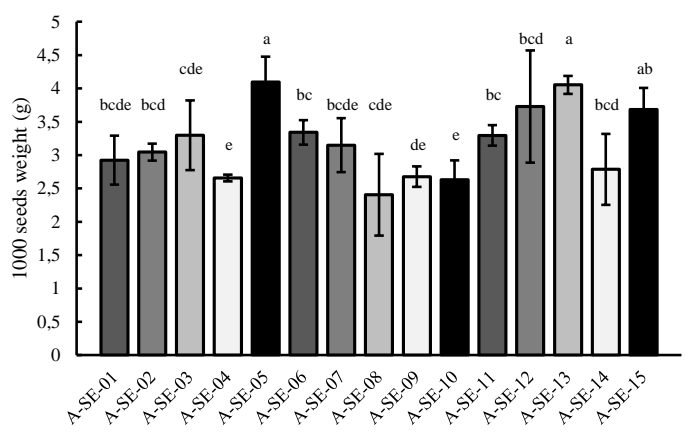

B

\subsection{Germination rates and seed viability}

To evaluate the germination capacity of the seeds, germination rates were determined for all cultivars harvested (Figure 5A). Noticeably differences were found in the germination rates of the various cultivars analyzed. Thus, while A-SE- $04 \mathrm{cv}$. and A-SE-15 $\mathrm{cv}$. showed germination rates above $50 \% 3$ d.a.s, reaching A-SE- $04 \mathrm{cv}$. 80\% germination rate 7 d.a.s (Figure 5B), A-SE-03 seeds were unable to germinate, and A-SE-06 cv. and ASE- $01 \mathrm{cv}$. did not overtake $20 \%$ germination rates 3 d.a.s. On the other hand, A-SE- $12 \mathrm{cv}$., although showing a germination delay, were able to reach almost $50 \%$ in the germinating rates 7 d.a.s, being close to the A-SE- $05 \mathrm{cv}$. germination rates at 7 d.a.s.

Seed viability was determined to complete the physiological analysis of the seeds (Figure5 C). For most of the cultivars, except for A-SE-15 cv., A-SE-04 cv., A-SE-03 cv. and A-SE-06 cv., seed viability showed no correlation with seed germination being, generally, severely reduced in most of the seeds tested. 


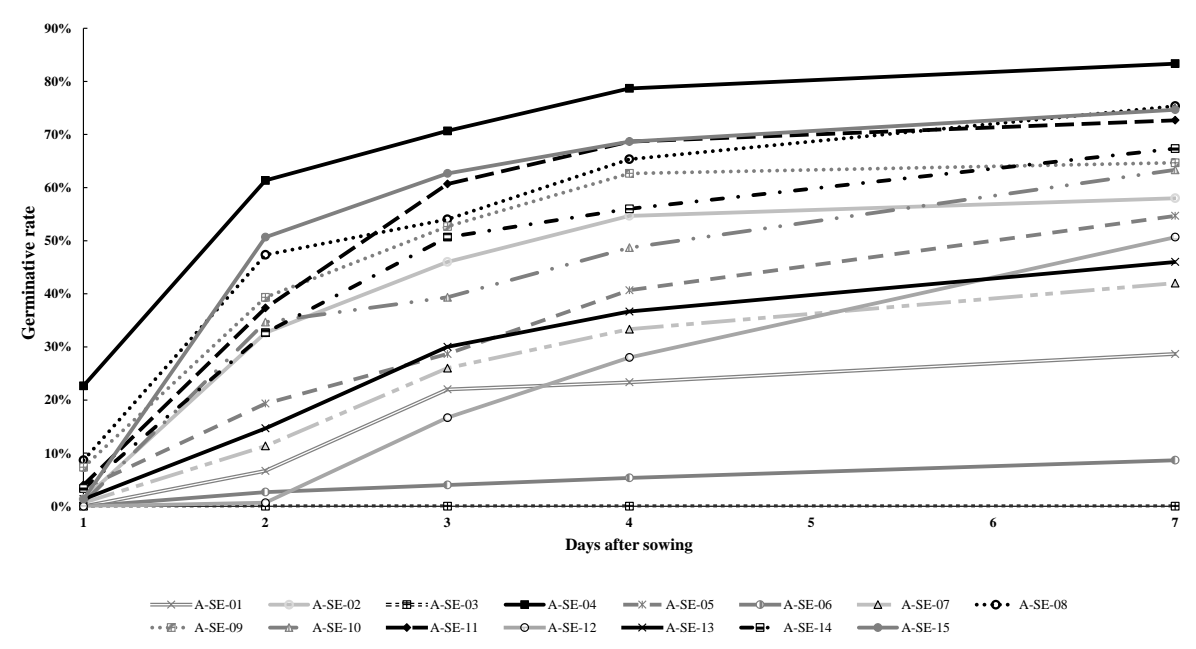

B

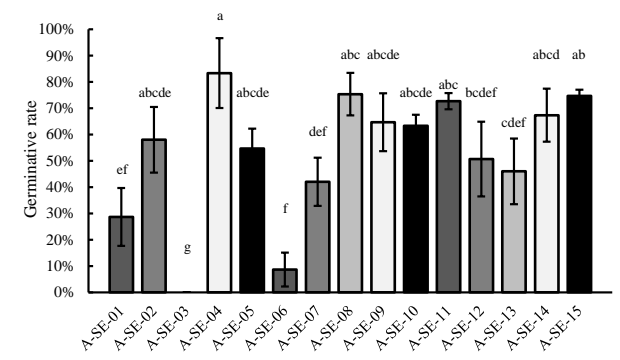

C

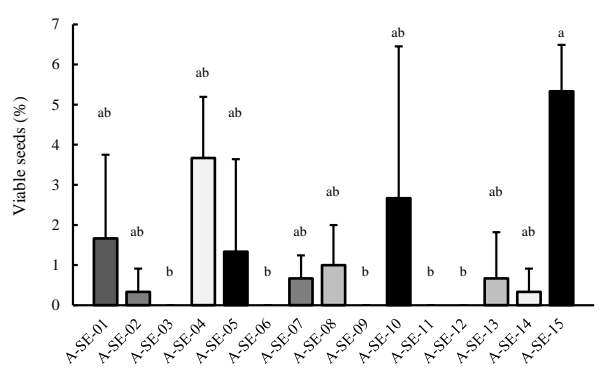

Figure 5. Germination rates (\%) and seed viability. A) Time course of germination percentage (\%) of quinoa seeds 1, 2, 3, 4, 5, 6 and 7 seven days after sowing (d.a.s.), B) Germination rate percentage (\%) 7 d.a.s. and C) Percentage (\%) of viable seeds. Error bars represent the standard deviation. Bars that do not share the same letters show statistically significant differences following Krustal-Wallis test by ranks for multiple comparisons at a $p$-value $<0.05$

\subsection{Protein content.}

Total protein contents in seeds revealed variations among cultivars (Figure 6). The cultivars A-SE- $15 \mathrm{cv}$. and A-SE-02 cv. showed the highest contents, and, in contrast, A-SE$06 \mathrm{cV}$. and A-SE-03 cv. showed the lowest. A gradient in total protein content was found as follows: A-SE-15 cv. $>$ (A-SE-02 cv.=A-SE-08 cv.=A-SE-12 cv. $)>(\mathrm{A}-\mathrm{SE}-10 \mathrm{cv} .=\mathrm{A}-\mathrm{SE}-11$ cv. $)>($ A-SE-0 4 cv. $=A-S E-13$ cv. $=$ A-SE-14 cv. $)>($ EM $1 \mathrm{cv} .=A-S E-05$ cv. $=A-S E-07$ cv. $=A-S E-09$ cV.) $>$ A-SE-03 cv.>A-SE-06 cv. Interestingly, as shown in Supplementary Figure 3, protein content positively correlated with TPC, FRAP, saponin content, $\mathrm{Zn}, \mathrm{Mg}$, and P and, negatively, with $\mathrm{C} / \mathrm{N}$ ratio and $\mathrm{Na}$ content. Regarding the physiological parameters, protein content correlated negatively with panicle height and panicle biomass and positively with the germination rate (Supplementary Figure 3). 


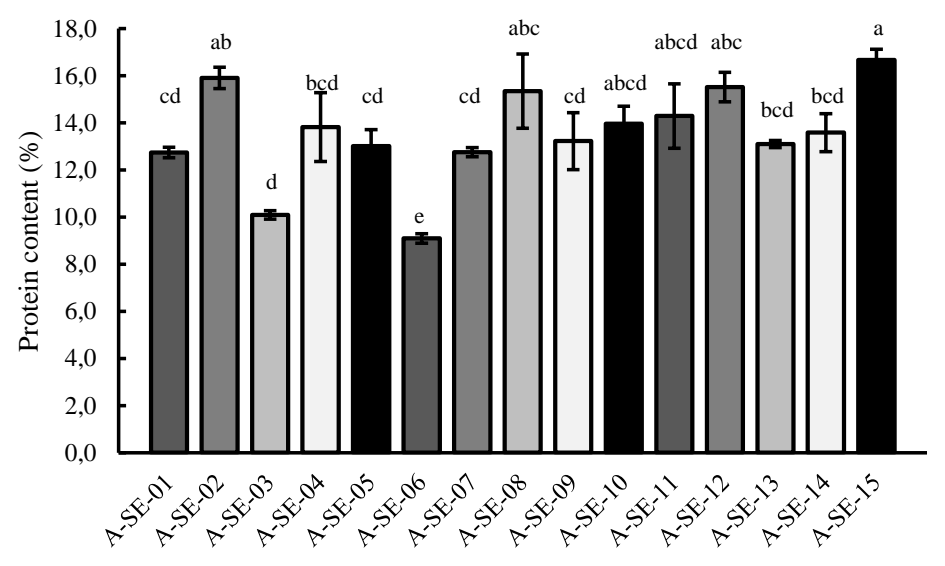

Figure 6. Seed protein content (\%). Protein content was determined in seeds of the 15 cultivars evaluated. Error bars represent the standard deviation. Bars that do not share the same letters show statistically significant differences following the Krustal-Wallis test by ranks for multiple comparisons at a $p$-value $<0.05$

\subsection{Mineral content.}

The total contents (as \%) of phosphorous $(\mathrm{P})$, potassium $(\mathrm{K})$, calcium $(\mathrm{Ca})$, magnesium $(\mathrm{Mg})$, (and as $\mathrm{mg} / \mathrm{Kg}$ ) sodium $(\mathrm{Na})$, copper $(\mathrm{Cu})$, iron $(\mathrm{Fe})$, manganese $(\mathrm{Mn})$, and zinc $(\mathrm{Zn})$ in quinoa seeds were determined to analyze the effect of the genotype on this nutritional-related parameter (Table 1). Some mineral nutrients such as Mg did not show significant variation among genotypes or, as in the case of $\mathrm{K}$, showed a small fluctuation. On the contrary, minerals such as $\mathrm{Zn}$, showed a steeper variation, being, from the highest to the lowest, A-SE-12 cv. the one with a higher content followed by A-SE-15 cv.>A-SE-13 cv. $>$ (A-SE-05 cv. =A-SE-08 cv.)>A-SE-07 cv.>A-SE-04 cv. >A-SE-02 cv.>(EM $1 \mathrm{cv} .=A-S E-14$ cv.) $>$ A-SE-11 cv. $>$ A-SE-06 cv.>A-SE-10 cv. $>$ A-SE-03 cv. $=$ A-SE-09 cv. Among cultivars, it should be noted that A-SE-12 cv. presented higher contents of $\mathrm{P}, \mathrm{Ca}, \mathrm{Fe}$, and $\mathrm{Zn}$, and intermediate levels of the rest of minerals, and A-SE-15 cv. presented the highest contents of $\mathrm{P}, \mathrm{Cu}$, and $\mathrm{Zn}$, and the lowest of $\mathrm{Ca}, \mathrm{Na}$, and Fe. At the same time, A-SE-03 cv., A-SE$04 \mathrm{cv}$., and A-SE-06 cv., had higher contents of $\mathrm{Ca}$ and $\mathrm{Na}$ and lower of $\mathrm{P}$ and $\mathrm{Cu}$.

Table 1. Mineral seed contents. Mean $\pm S D$ mineral contents are presented as percentage of seed weight $(\mathrm{P}, \mathrm{K}, \mathrm{Ca}$, and $\mathrm{Mg}$ ) or as $\mathrm{mg} / \mathrm{Kg}(\mathrm{Na}, \mathrm{Fe}, \mathrm{Cu}, \mathrm{Mn}$, and $\mathrm{Zn})$. Statistical analysis following a Krustal-Wallis test by ranks ( $\mathrm{P}, \mathrm{K}, \mathrm{Mg}, \mathrm{Na}, \mathrm{Mn}, \mathrm{Cu}, \mathrm{Zn}$ content) or a Welch's ANOVA with a Games-Howell post-hoc test (Ca and Fe contents) was performed. Different letters under each mineral content show statistically significant differences between samples. 


\begin{tabular}{|c|c|c|c|c|c|c|c|c|c|c|c|c|}
\hline & Ash (\%) & litrogen $(\%)$ & $\mathrm{C} / \mathrm{N}$ ratio & $P(\%)$ & K (\%) & $\mathrm{Ca}(\%)$ & $\operatorname{Mg}(\%)$ & $\mathrm{Na}$ (ppm) & $\mathrm{Fe}$ (ppm) & Mn (ppm) & Cu (ppm) & Zn (ppm) \\
\hline A-SE-01 & $\begin{array}{c}3.48 \pm 0.02 \\
b\end{array}$ & $\begin{array}{c}2.04 \pm 0.04 \\
\text { cd }\end{array}$ & $\begin{array}{c}18.45 \pm 0.35 \\
\text { bc }\end{array}$ & $\begin{array}{l}29 \pm 0.00 \\
\text { bc }\end{array}$ & $\begin{array}{l}14 \pm 0.01 \\
\mathrm{ab}\end{array}$ & $\begin{array}{c}0.30 \pm 0.02 \\
\text { abc }\end{array}$ & $\begin{array}{c}.21 \pm 0.00 \\
-\end{array}$ & $\begin{array}{c}146.98 \pm 5.99 \\
\text { bcdefg }\end{array}$ & $\begin{array}{c}40.41 \pm 0.27 \\
b\end{array}$ & $\begin{array}{c}19.57 \pm 0.30 \\
\text { bcdef }\end{array}$ & $\begin{array}{c}9.30 \pm 1.42 \\
\text { bcde }\end{array}$ & $\begin{array}{l}29.18 \pm 0.34 \\
\text { efg }\end{array}$ \\
\hline A-SE-02 & $\begin{array}{c}3.22 \pm 0.10 \\
\text { abc }\end{array}$ & $\begin{array}{c}2.55 \pm 0.07 \\
\mathrm{ab}\end{array}$ & $\begin{array}{c}14.81 \pm 0.56 \\
\mathrm{de}\end{array}$ & $\begin{array}{c}0.27 \pm 0.00 \\
\text { bc }\end{array}$ & $\begin{array}{c}1.03 \pm 0.01 \\
\mathrm{~b}\end{array}$ & $\begin{array}{c}0.28 \pm 0.01 \\
\text { bc }\end{array}$ & $\begin{array}{c}19 \pm 0.01 \\
-\end{array}$ & $\begin{array}{c}116.63 \pm 7.66 \\
\mathrm{gh}\end{array}$ & $\begin{array}{c}38.98 \pm 1.72 \\
\mathrm{~b}\end{array}$ & $\begin{array}{c}11.69 \pm 0.05 \\
\mathrm{f}\end{array}$ & $\begin{array}{c}11.70 \pm 1.73 \\
\text { bc }\end{array}$ & $\begin{array}{c}29.56 \pm 0.44 \\
\text { ef }\end{array}$ \\
\hline A-SE-03 & $\begin{array}{c}3.47 \pm 0.07 \\
\text { abc }\end{array}$ & $\begin{array}{c}1.62 \pm 0.03 \\
\mathrm{~d}\end{array}$ & $\begin{array}{c}22.59 \pm 0.28 \\
\mathrm{~b}\end{array}$ & $\begin{array}{c}0.17 \pm 0.00 \\
\mathrm{~d}\end{array}$ & $\begin{array}{c}1.26 \pm 0.01 \\
\mathrm{ab}\end{array}$ & $\begin{array}{c}0.38 \pm 0.02 \\
\mathrm{a}\end{array}$ & $\begin{array}{c}0.19 \pm 0.00 \\
-\end{array}$ & $\begin{array}{c}233.42 \pm 55.08 \\
a b\end{array}$ & $\begin{array}{c}36.05 \pm 1.35 \\
\mathrm{~b}\end{array}$ & $\begin{array}{c}16.65 \pm 0.99 \\
\text { cdef }\end{array}$ & $\begin{array}{c}7.54 \pm 0.56 \\
\text { cdef }\end{array}$ & $\begin{array}{c}23.93 \pm 0.38 \\
\mathrm{i}\end{array}$ \\
\hline A-SE-04 & $\begin{array}{c}3.03 \pm 0.33 \\
\mathrm{abc}\end{array}$ & $\begin{array}{c}2.21 \pm 0.23 \\
\text { bcd }\end{array}$ & $\begin{array}{l}17.47 \pm 1.56 \\
\text { bcd }\end{array}$ & $\begin{array}{l}0.22 \pm 0.02 \\
\mathrm{~cd}\end{array}$ & $\begin{array}{c}0.98 \pm 0.12 \\
\mathrm{ab}\end{array}$ & $\begin{array}{c}0.35 \pm 0.02 \\
a b\end{array}$ & $\begin{array}{c}0.20 \pm 0.02 \\
-\end{array}$ & $\begin{array}{c}279.7 \pm 37.63 \\
\mathrm{a}\end{array}$ & $\begin{array}{c}32.85 \pm 3.45 \\
\mathrm{bc}\end{array}$ & $\begin{array}{c}13.83 \pm 0.95 \\
\text { ef }\end{array}$ & $\begin{array}{c}7.86 \pm 1.21 \\
\text { bcdef }\end{array}$ & $\begin{array}{c}29.95 \pm 0.52 \\
\text { def }\end{array}$ \\
\hline A-SE-05 & $\begin{array}{c}3.66 \pm 0.27 \\
\text { abc }\end{array}$ & $\begin{array}{c}2.08 \pm 0.11 \\
\mathrm{~cd}\end{array}$ & $\begin{array}{c}18.21 \pm 0.92 \\
\text { bcd }\end{array}$ & $\begin{array}{c}0.27 \pm 0.02 \\
\text { bcd }\end{array}$ & $\begin{array}{c}1.24 \pm 0.10 \\
\mathrm{ab}\end{array}$ & $\begin{array}{c}0.35 \pm 0.04 \\
\text { abcd }\end{array}$ & $\begin{array}{c}0.18 \pm 0.01 \\
-\end{array}$ & $\begin{array}{c}173.49 \pm 21.92 \\
\text { abcdef }\end{array}$ & $\begin{array}{c}29.73 \pm 2.74 \\
\text { bc }\end{array}$ & $\begin{array}{c}13.42 \pm 0.72 \\
\text { def }\end{array}$ & $\begin{array}{c}9.34 \pm 0.80 \\
\text { bcde }\end{array}$ & $\begin{array}{c}31.60 \pm 0.74 \\
\text { bed }\end{array}$ \\
\hline A-SE-06 & $\begin{array}{c}3.11 \pm 0.17 \\
\text { abc }\end{array}$ & $\begin{array}{c}1.46 \pm 0.03 \\
\mathrm{e}\end{array}$ & $\begin{array}{c}25.34 \pm 0.44 \\
\mathrm{a}\end{array}$ & $\begin{array}{c}0.15 \pm 0.00 \\
\mathrm{e}\end{array}$ & $\begin{array}{c}1.13 \pm 0.03 \\
\mathrm{ab}\end{array}$ & $\begin{array}{c}0.36 \pm 0.12 \\
\text { abcdef }\end{array}$ & $\begin{array}{c}0.17 \pm 0.02 \\
-\end{array}$ & $\begin{array}{c}194.52 \pm 59.36 \\
\text { abcde }\end{array}$ & $\begin{array}{c}35.08 \pm 15.60 \\
\text { bc }\end{array}$ & $\begin{array}{c}14.32 \pm 6.60 \\
\text { cdef }\end{array}$ & $\begin{array}{c}9.40 \pm 3.17 \\
\text { bcdef }\end{array}$ & $\begin{array}{c}25.53 \pm 1.23 \\
\text { ghi }\end{array}$ \\
\hline A-SE-07 & $\begin{array}{c}3.18 \pm 0.09 \\
\text { bc }\end{array}$ & $\begin{array}{c}2.04 \pm 0.03 \\
\mathrm{~cd}\end{array}$ & $\begin{array}{c}18.40 \pm 0.45 \\
\text { bc }\end{array}$ & $\begin{array}{l}0.26 \pm 0.01 \\
\text { bcd }\end{array}$ & $\begin{array}{c}1.10 \pm 0.02 \\
\mathrm{ab}\end{array}$ & $\begin{array}{c}0.18 \pm 0.02 \\
\text { def }\end{array}$ & $\begin{array}{c}0.19 \pm 0.00 \\
-\end{array}$ & $\begin{array}{l}\begin{array}{c}125.43 \pm 14.68 \\
\text { efgh }\end{array}\end{array}$ & $\begin{array}{c}42.71 \pm 3.77 \\
\text { abc }\end{array}$ & $\begin{array}{l}19.92 \pm 0.18 \\
\text { bcde }\end{array}$ & $\begin{array}{c}10.28 \pm 0.31 \\
\text { bc }\end{array}$ & $\begin{array}{l}30.85 \pm 0.22 \\
\text { cde }\end{array}$ \\
\hline A-SE-08 & $\begin{array}{c}3.43 \pm 0.36 \\
a b c\end{array}$ & $\begin{array}{c}2.46 \pm 0.25 \\
\text { abc }\end{array}$ & $\begin{array}{c}15.65 \pm 1.65 \\
\text { bcde }\end{array}$ & $\begin{array}{c}0.28 \pm 0.03 \\
\text { bc }\end{array}$ & $\begin{array}{c}1.15 \pm 0.12 \\
\mathrm{ab}\end{array}$ & $0.21 \pm 0.02$ & $0.22 \pm 0.02$ & $141.79 \pm 12.98$ & $\begin{array}{c}43.18 \pm 3.60 \\
\text { abc }\end{array}$ & $22.90 \pm 0.38$ & $10.32 \pm 0.44$ & $\begin{array}{c}31.28 \pm 0.80 \\
\text { bed }\end{array}$ \\
\hline A-SE-09 & $\begin{array}{c}3.50 \pm 0.47 \\
\mathrm{abc}\end{array}$ & $\begin{array}{c}2.12 \pm 0.19 \\
\mathrm{~cd}\end{array}$ & $\begin{array}{l}17.90 \pm 1.50 \\
\text { bcd }\end{array}$ & $\begin{array}{c}0.26 \pm 0.03 \\
\text { bcd }\end{array}$ & $\begin{array}{c}1.16 \pm 0.16 \\
a b\end{array}$ & $\begin{array}{c}0.27 \pm 0.01 \\
\text { bcde }\end{array}$ & $\begin{array}{c}0.18 \pm 0.02 \\
-\end{array}$ & $\begin{array}{c}153.07 \pm 32.93 \\
\text { bcdefgh }\end{array}$ & $\begin{array}{c}39.54 \pm 4.68 \\
\text { abc }\end{array}$ & $\begin{array}{c}22.19 \pm 1.50 \\
\text { ab }\end{array}$ & $\begin{array}{c}7.08 \pm 0.53 \\
\text { def }\end{array}$ & $\begin{array}{c}23.80 \pm 0.25 \\
\text { i }\end{array}$ \\
\hline A-SE-10 & $\begin{array}{c}3.68 \pm 0.34 \\
\text { abc }\end{array}$ & $\begin{array}{c}2.24 \pm 0.12 \\
\text { abcd }\end{array}$ & $\begin{array}{c}16.95 \pm 0.99 \\
\text { bcde }\end{array}$ & $\begin{array}{l}0.24 \pm 0.04 \\
\text { bcd }\end{array}$ & $\begin{array}{c}1.22 \pm 0.17 \\
\mathrm{ab}\end{array}$ & $\begin{array}{c}0.25 \pm 0.04 \\
\text { abcdef }\end{array}$ & $\begin{array}{c}0.19 \pm 0.03 \\
-\end{array}$ & $\begin{array}{c}191.24 \pm 21.87 \\
\text { abcd }\end{array}$ & $\begin{array}{c}47.12 \pm 8.56 \\
\text { abc }\end{array}$ & $\begin{array}{l}20.77 \pm 2.11 \\
\text { abcd }\end{array}$ & $\begin{array}{c}6.41 \pm 1.01 \\
\text { ef }\end{array}$ & $\begin{array}{c}25.32 \pm 1.36 \\
\mathrm{hi}\end{array}$ \\
\hline A-SE-11 & $\begin{array}{c}3.62 \pm 0.63 \\
\text { abc }\end{array}$ & $\begin{array}{c}2.29 \pm 0.22 \\
\text { abcd }\end{array}$ & $\begin{array}{c}16.74 \pm 1.57 \\
\text { bcde }\end{array}$ & $\begin{array}{c}0.28 \pm 0.03 \\
\text { bcd }\end{array}$ & $\begin{array}{c}1.23 \pm 0.21 \\
\mathrm{ab}\end{array}$ & $\begin{array}{c}0.24 \pm 0.03 \\
\text { abcdef }\end{array}$ & $\begin{array}{c}0.21 \pm 0.03 \\
-\end{array}$ & $\begin{array}{c}194.76 \pm 22.11 \\
\text { abc }\end{array}$ & $\begin{array}{c}39.56 \pm 6.24 \\
\text { abc }\end{array}$ & $\begin{array}{c}20.05 \pm 1.63 \\
\text { abcd }\end{array}$ & $\begin{array}{c}6.55 \pm 0.68 \\
\mathrm{f}\end{array}$ & $\begin{array}{c}28.76 \pm 0.30 \\
\text { fgh }\end{array}$ \\
\hline A-SE-12 & $\begin{array}{c}3.26 \pm 0.03 \\
c\end{array}$ & $\begin{array}{c}2.48 \pm 0.10 \\
\text { abc }\end{array}$ & $\begin{array}{c}15.13 \pm 0.59 \\
\text { cde }\end{array}$ & $\begin{array}{c}0.31 \pm 0.01 \\
\mathrm{~b}\end{array}$ & $\begin{array}{c}1.12 \pm 0.01 \\
\mathrm{ab}\end{array}$ & $\begin{array}{c}0.36 \pm 0.01 \\
\mathrm{a}\end{array}$ & $\begin{array}{c}0.19 \pm 0.00 \\
-\end{array}$ & $\begin{array}{c}139.22 \pm 16.03 \\
\text { defgh }\end{array}$ & $\begin{array}{c}56.39 \pm 1.56 \\
\mathrm{a}\end{array}$ & $\begin{array}{c}17.62 \pm 0.51 \\
\text { cdef }\end{array}$ & $\begin{array}{c}8.98 \pm 0.76 \\
\text { bcdef }\end{array}$ & $\begin{array}{c}35.23 \pm 0.21 \\
\mathrm{a}\end{array}$ \\
\hline A-SE-13 & $\begin{array}{c}3.73 \pm 0.03 \\
\mathrm{a}\end{array}$ & $\begin{array}{c}2.10 \pm 0.02 \\
\text { bcd }\end{array}$ & $\begin{array}{c}17.93 \pm 0.28 \\
\text { bcd }\end{array}$ & $\begin{array}{c}0.27 \pm 0.01 \\
\text { bcd }\end{array}$ & $\begin{array}{c}1.39 \pm 0.03 \\
\mathrm{a}\end{array}$ & $\begin{array}{c}0.10 \pm 0.03 \\
\text { ef }\end{array}$ & $\begin{array}{c}0.18 \pm 0.01 \\
-\end{array}$ & $\begin{array}{c}121.64 \pm 15.62 \\
\text { fgh }\end{array}$ & $\begin{array}{c}35.17 \pm 3.55 \\
\mathrm{bc}\end{array}$ & $\begin{array}{c}22.17 \pm 1.15 \\
\text { abc }\end{array}$ & $\begin{array}{c}19.52 \pm 0.60 \\
\mathrm{a}\end{array}$ & $\begin{array}{c}32.45 \pm 0.58 \\
\text { bc }\end{array}$ \\
\hline A-SE-14 & $\begin{array}{c}3.34 \pm 0.21 \\
\mathrm{abc}\end{array}$ & $\begin{array}{c}2.17 \pm 0.13 \\
\text { bcd }\end{array}$ & $\begin{array}{c}17.52 \pm 1.00 \\
\text { bcd }\end{array}$ & $\begin{array}{l}0.25 \pm 0.01 \\
\text { bcd }\end{array}$ & $\begin{array}{c}1.16 \pm 0.06 \\
\mathrm{ab}\end{array}$ & $\begin{array}{c}0.10 \pm 0.02 \\
f\end{array}$ & $\begin{array}{c}0.20 \pm 0.01 \\
-\end{array}$ & $\begin{array}{c}125.35 \pm 15.11 \\
\text { fgh }\end{array}$ & $\begin{array}{c}33.19 \pm 2.41 \\
\mathrm{bc}\end{array}$ & $\begin{array}{c}23.16 \pm 1.16 \\
\mathrm{a}\end{array}$ & $\begin{array}{c}18.46 \pm 0.88 \\
\mathrm{ab}\end{array}$ & $\begin{array}{c}29.37 \pm 0.97 \\
\text { efg }\end{array}$ \\
\hline 15 & $\begin{array}{c}3.19 \pm 0.08 \\
\text { bc }\end{array}$ & $\begin{array}{c}2.67 \pm 0.07 \\
\mathrm{a}\end{array}$ & $\begin{array}{c}14.39 \pm 0.48 \\
\mathrm{e}\end{array}$ & $\begin{array}{c}0.35 \pm 0.01 \\
\mathrm{a}\end{array}$ & $\begin{array}{c}1.12 \pm 0.02 \\
a b\end{array}$ & $\begin{array}{c}0.09 \pm 0.01 \\
\mathrm{f}\end{array}$ & $\begin{array}{c}0.21 \pm 0.01 \\
-\end{array}$ & $\begin{array}{c}100.87 \pm 8.96 \\
\mathrm{~h}\end{array}$ & $\begin{array}{c}25.10 \pm 1.29 \\
\mathrm{c}\end{array}$ & $\begin{array}{c}20.34 \pm 0.50 \\
\text { abcd }\end{array}$ & $\begin{array}{c}19.75 \pm 0.96 \\
\mathrm{a}\end{array}$ & $\begin{array}{c}34.09 \pm 0.81 \\
\mathrm{~b}\end{array}$ \\
\hline
\end{tabular}

\subsection{Antioxidant capacity.}

We evaluated the antioxidant capacity of the seeds by performing the FRAP assay and the quantification of total polyphenols (TPC) and flavonoids (TFC) contents (Figure 7). Among cultivars, A-SE- $04 \mathrm{cv}$. followed by A-SE- $15 \mathrm{cv}$. showed the highest antioxidant capacity, presenting high values of FRAP, TPC, and TFC levels. On the contrary, A-SE-01 cv. together with A-SE-06 cv. the lowest. The other cultivars showed distinct patterns, presenting changes among the antioxidant-related parameters here evaluated. For instance, A-SE-10 cv. showed intermediate and high FRAP and TFC values, respectively, and low TPC levels. 
A

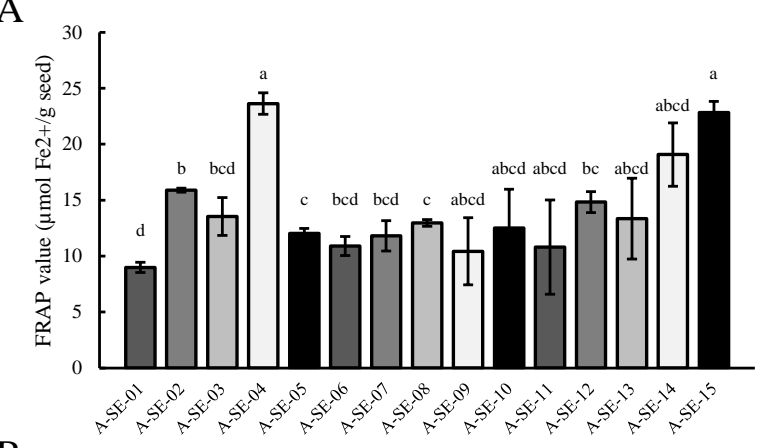

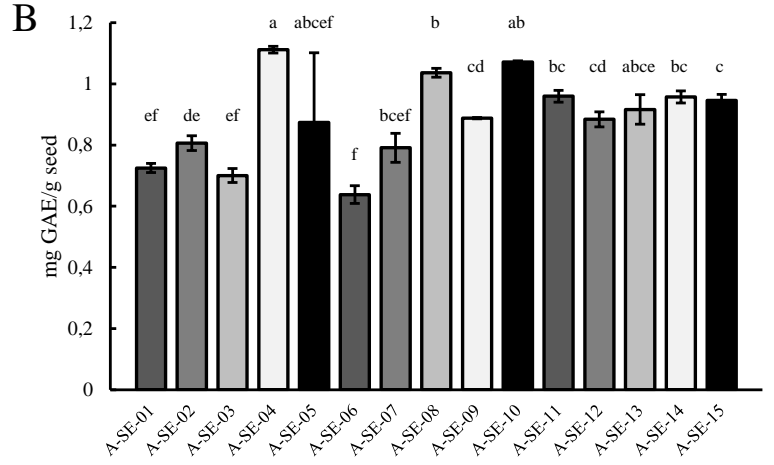

C

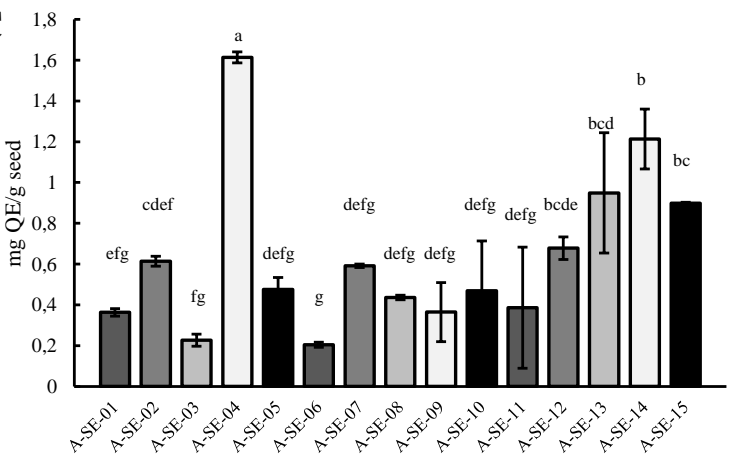

Figure 7. Antioxidant capacity of quinoa seeds. A) Antioxidant power determined by FRAP assay and is expressed as $\mu \mathrm{mol}$ of $\mathrm{Fe} 2+$ per gram of seed. Statistical differences were analyzed through a Welch's ANOVA test followed by a Games-Howell post-hoc test. B) TPC is expressed as milligrams of gallic acid equivalents (GAE) per gram of seeds. The statistical analysis performed was a Welch's ANOVA test followed by a Games-Howell post-hoc test. C) TFC is expressed as milligrams of quercetin equivalents (QE) per gram of seeds. A Krustal-Wallis test by ranks was performed for multiple comparisons. Bars that do not share the same letters show statistically significant differences at a $p$-value $<0.05$. Error bars represent the standard deviation.

\subsection{Saponin content}

Saponin content was quantified in the cultivars studied (Figure 8). A-SE-06 was the cultivar showing the lowest saponin content while A-SE-10 was the cultivar with the highest saponin level. All of the cultivars exceed the limit of $0.11 \%$ established to classify quinoa varieties as sweet [27], however, none of them presented a content higher than $1 \%$, which is usually overtaken by bitter quinoa seeds [28]. 


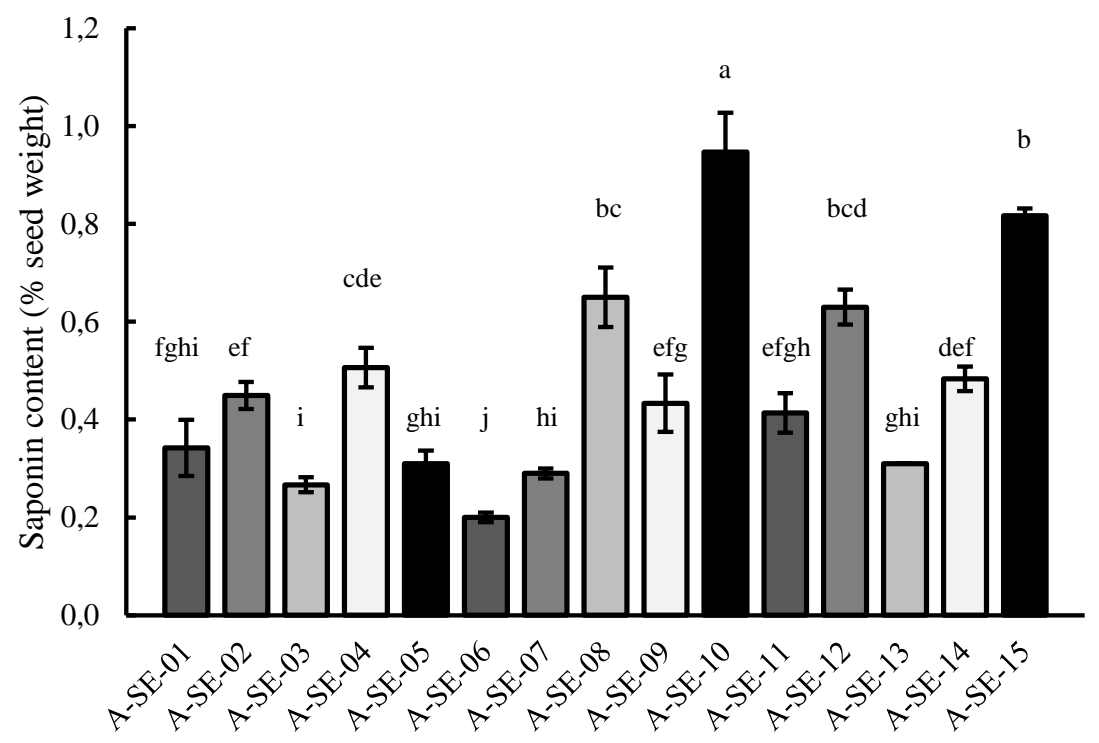

Figure 8. Saponin content. Saponin content was determined in seeds of the 15 cultivars evaluated. Error bars represent the standard deviation. Bars that do not share the same letters show statistically significant differences following the Krustal-Wallis test by at a $p$-value $<0.05$.

\subsection{Path coefficient analysis}

Path analysis was performed to define the direct and indirect contributions of each trait on seed yield. First, a predictive multiple linear regression model was performed following the stepwise method in order to find physiological traits with a direct effect on germination rates (Table 2) and yield (Table 3). As shown in Table 2, germination rates would be affected positively by the phenols (TPC) and P contents and indirectly by physiological parameters such as seed area or panicle biomass or biochemical properties of seeds such as protein or saponin content. On the other hand, yield would be explained in a negative way by the seed weight and panicle height, and positively by the panicle biomass and total biomass, meaning that these parameters may directly impact the seed yield of the quinoa varieties here analyzed. 
Table 2 Direct effects of predictor variables of first-, second-, third-, and fourth-order on germination rate, tolerance, and variance inflation factor of the path analysis. Subsequent multiple linear regression analysis was performed. TPC: total phenol content. P: total phosphorous and Mg: total magnesium.

\begin{tabular}{cccccc} 
Response variable & Predictor varibles & Adjusted $\mathrm{R}^{2}$ & Direct effect & Tolerance & VIF \\
\hline Germination & TPC & 0,825 & 0,750 & 0,947 & 1,056 \\
& $\mathrm{P}$ & & 0,390 & 0,947 & 1,056 \\
\hline TPC & Saponins & 0,723 & 0,584 & 0,838 & 1,193 \\
& Seed Area & & $-0,456$ & 0,838 & 1,193 \\
\hline Saponins & Protein & 0,876 & 0,731 & 0,781 & 1,280 \\
& Panicle Biomass & & $-0,636$ & 0,496 & 2,016 \\
& Panicle Height & & 0,844 & 0,572 & 1,749 \\
\hline Protein & Mg & 0,273 & 0,570 & 1 & 1 \\
\hline Panicle Biomass & Biomass & 0,558 & 0,602 & 1 & 1 \\
& Mildew damage (128 das) & & 0,521 & 1 & 1 \\
\hline P & Panicle Height & 0,738 & $-0,371$ & 0,901 & 1,110
\end{tabular}

Table 3. Direct effects of predictor variables of first-, second-, third-, and fourth-order yield, tolerance, and variance inflation factor of the path analysis. Subsequent multiple linear regression analysis was performed.

\begin{tabular}{cccccc}
$\begin{array}{c}\text { Response } \\
\text { variable }\end{array}$ & Predictor varibles & $\begin{array}{c}\text { Adjusted } \\
\mathrm{R}^{2}\end{array}$ & $\begin{array}{c}\text { Direct } \\
\text { effect }\end{array}$ & Tolerance & VIF \\
\hline Yield & Biomass & 0,880 & 0,481 & 0,624 & 1,603 \\
& Panicle Biomass & & 0,469 & 0,522 & 1,914 \\
& Seed Weight & & $-0,405$ & 0,975 & 1,026 \\
& Panicle Height & & $-0,102$ & 0,631 & 1,584 \\
\hline Biomass & Plant Height (128 das) & 0,013 & $-0,124$ & 1 & 1 \\
\hline Seed Weight & Plant Height (128 das) & 0,035 & 0,173 & 0,999 & 1,001 \\
& Mildew damage (128 das) & & $-0,109$ & 0,999 & 1,001 \\
\hline Panicle Height & Plant Height (128 das) & 0,106 & $-0,194$ & 0,999 & 1,001 \\
& Mildew severity (128 das) & & 0,277 & 0,999 & 1,001 \\
\hline Panicle & Plant Height (128 das) & \multirow{2}{*}{0,061} & $-0,121$ & 0,999 & 1,001 \\
Biomass & Mildew severity (128 das) & & 0,230 & 0,999 & 1,001
\end{tabular}

\subsection{Principal components analysis $(P C A)$}

A Pearson's correlation coefficient test was performed to analyze the correlation between variables (Supplementary figure 3) and a principal component analysis (PCA) to reduce the number of variables. This analysis identified five principal components that were able to explain $74.76 \%$ of the variance. Component 1 , which contributes to $21.31 \%$ of the variance, was mainly explained by the protein and saponin contents and most minerals contents ( $\mathrm{P}, \mathrm{Ca}, \mathrm{Mg}, \mathrm{Mn}, \mathrm{Cu}$, and $\mathrm{Zn}$ contribute positively, and $\mathrm{Ca}$ and Na negatively), and by the germination rate, lodging, and plant height and mildew severity at 128 d.a.s. 
For this new variable, A-SE-12 cv. and A-SE-15 cv. show high values while A-SE-03 cv. and A-SE-06 cv. present the lowest. There were correlations between most of these variables, but those between protein content and germinative rate $(\mathrm{r}=0.801)$, protein and $\mathrm{P}$ contents $(\mathrm{r}=0.846), \mathrm{P}$ and $\mathrm{Zn}$ contents $(\mathrm{r}=0.728)$, and protein and saponin contents $(\mathrm{r}=0.695)$ were the strongest (Figure 9). Component 2 contributes to variance with $18.30 \%$, and comprises panicle length and biomass, plant dry weight, yield, germinative rate of seeds, and total phenolic content, and inversely plant height (at three time points) and seed area. Plant height at early stages (82 and $100 \mathrm{~d}$. a. s.) correlates negatively with the final plant biomass, yield, germinative rate of seeds, and protein and phenolic contents, while the panicle height and weight at $128 \mathrm{~d}$. a. s. correlates positively with these parameters. Strong correlations are found between the phenolic content and the germinative rate and panicle height ( $\mathrm{r}=0.884$ and $\mathrm{r}=0,780$, respectively). For this component, there are high values in ASE-08 cv. and A-SE-04 cv. and low in A-SE-01 cv. Component 3 explains a 12.19\% of variance and comprises the viability rate, flavonoid contents, and antioxidant capacity, and inversely ash, $\mathrm{K}$, and Fe contents. Both viability and germinative rate correlate with each other and with the antioxidant capacity and phenolic and flavonoid contents. There is also a strong correlation between ash and $\mathrm{K}$ content $(\mathrm{r}=0.851)$ since $\mathrm{K}$ is the main mineral present in quinoa (Table 1). A-SE-04 cv. and A-SE- $15 \mathrm{cv}$. show high component 3 values and A-SE-09 cv., A-SE-11 cv., and A-SE-13 show low values. Area and seed weight, $\mathrm{K}$ and $\mathrm{Cu}$ content contribute positively to component 4 (explaining $11.96 \%$ of variance) and $\mathrm{Fe}$ and $\mathrm{Ca}$ contents negatively. Area and seed weight show a strong correlation $(\mathrm{r}=0.748)$. A-SE$13 \mathrm{cv}$. presents the highest and A-SE-01 cv., A-SE- 09 cv., and A-SE- $10 \mathrm{cv}$. the lowest values for component 4 . Component 5 ( $11.00 \%$ of variance) comprises saponin content in seeds, panicle length, and mildew severity at three stages. There is a correlation between mildew severity at 82 and $100 \mathrm{~d}$. a. s., but not with severity at $128 \mathrm{~d}$. a. s., and saponin content and panicle height also show a strong correlation $(\mathrm{r}=0.655)$. A-SE-10 cv. showed high component 5 values while A-SE-12 cv. showed the lowest. Life cycle duration correlates with yield, the germinative rate of seeds, and their phenolic contents.

Plotting component 1 against component 2 reveals three clusters of cultivars (Figure 9). The first cluster is made up of A-SE-03 and A-SE-06 and is low for both components 1 and 2, which means they have taller plants at early stages but low yields, germinative capacity, and protein, $\mathrm{P}$, saponin, and phenolic contents. These cultivars are also low for component 3, and show the lowest viability rate, low antioxidant capacity but high ash content. The second cluster, comprising A-SE-01 cv., A-SE-02 cv., A-SE-07 cv., A-SE-12 cv., and A-SE-15 cv., has low component 2 values, especially A-SE-01 cv., so they are tall plants with smaller panicles, and lower yields, but higher component 1 values than the first cluster, so generally they have higher protein, $\mathrm{P}$, and $\mathrm{Cu}$ contents and lower $\mathrm{Ca}$ and $\mathrm{Na}$ contents than A-SE-03 cv. and A-SE-06. A-SE-05 cv., A-SE-08 cv., A-SE-09 cv., A-SE-10 cv., ASE-11 cv., A-SE-13 cv., and A-SE-14 cv. comprise the third cluster, which has high component 2 values, having shorter plants and larger panicles, heavier plants, higher yields and germination rates, and higher protein and phenolic contents. A-SE-04 cv. and A-SE-15 cv. show the highest component 3 values, with the largest viability rates and FRAP values, but A-SE-0 $4 \mathrm{cv}$. has lower component 1 values, with high $\mathrm{Ca}$ and $\mathrm{Na}$ contents and lower $\mathrm{Cu}$ and $\mathrm{Zn}$ contents, while A-SE-15 cv. has high component 1 values, and has high germination rates, and protein, $\mathrm{P}, \mathrm{Cu}$, and $\mathrm{Zn}$ contents and low $\mathrm{Ca}$ and $\mathrm{Na}$ contents. 


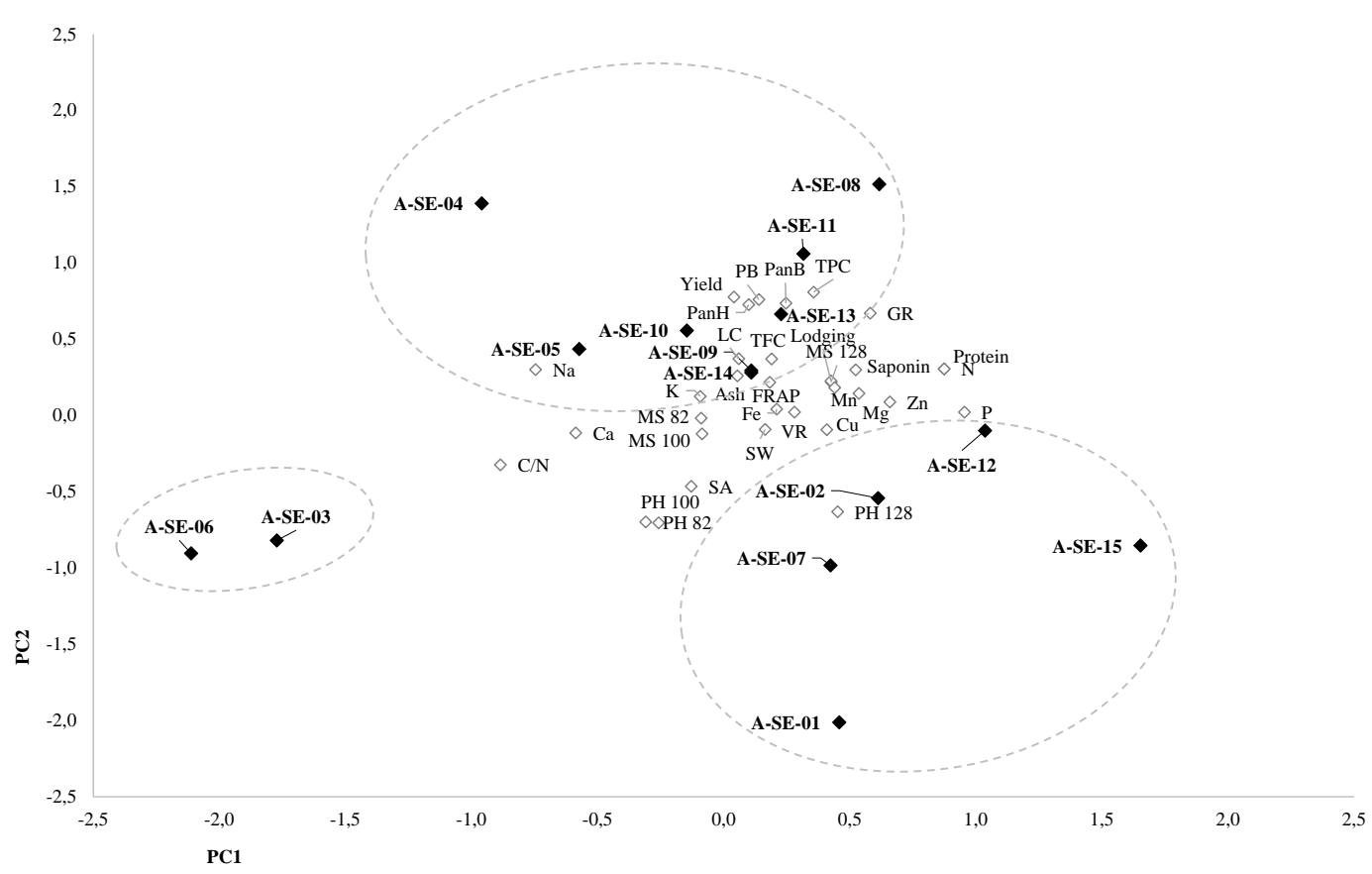

Figure 9. Principal components analysis (PCA). Biplot of main components 1 and 2 for the cultivars sown and for the variables tested. Component 1 (X-axis) is contributed mainly by : protein and saponin contents, germination rate, minerals $(\mathrm{P}, \mathrm{Mg}, \mathrm{Ca}(-), \mathrm{Na}(-), \mathrm{Mn}, \mathrm{Cu}, \mathrm{Zn})$, lodging, plant height 128 d.a.s., mildew 128 d.a.s. Component 2 (Y-axis) includes germination rate, total phenolics content, plant height (-), plant biomass, panicle height, and panicle weight (-).

\section{Discussion}

Quinoa is often compared to cereals and even considered a 'pseudocereal' due to the similarities in the composition and uses of their seeds [29]. It presents unique nutritional properties that make its cultivation very interesting [30]. However, its yield potential in new cultivation areas is yet far to be reached, with levels similar to those of cereals such as wheat or rice before the Green Revolution [2]. The center of origin of quinoa is the Andean Altiplano [31], but in the last decades, quinoa has been introduced as an alternative emerging crop in more than 75 countries [5]. Along with its expansion, it has been observed that the establishment and adaptation of quinoa cultivars to these new agroclimatic contexts can result in changes in the nutritional properties of quinoa seeds which are associated with variations in the genotype $(G)$, the environment $(E)$ and their interaction (GXE) [14, 21]. Thus, there is yet much left for researchers and breeders to do to develop quinoa cultivars better adapted to specific locations presenting high yields while also maintaining or even improving the nutritional value of the seeds. In this study, we evaluated the physiological characteristics and different nutritional-related traits of seeds harvested from 15 different quinoa cultivars grown in southern Spain, aiming to expand our knowledge of the relationship between yield and the nutritional quality of quinoa seeds and therefore, contributing to the selection of quinoa cultivars more appropriate for cultivation in a particular area of interest.

As previously described, the PCA revealed the existence of three clusters in which cultivars could be classified and showed distinct characteristics (Figure 9). At earlier stages of development (82 and 100 d. a. s.), plants from clusters 1 (A-SE-03 cv. and A-SE$06 \mathrm{cv}$.) and 2 (A-SE-01, A-SE-02 cv., A-SE-07 cv., and A-SE-15 cv.) were the tallest, but only those from cluster 2 kept taller at a later developmental stage (128 d. a. s., beginning of grain maturation) (Figure 1). Cluster 1 and cluster 2 plants also presented lower panicle lengths and biomass (Figure 2), and smaller seed yields. Seeds from these cultivars did not show higher nor lower seed weights or areas, and there was no correlation between 
these variables and yield (Supplementary Figure 3). From this, it can be assumed that plants from clusters 1 and 2, which invested more resources on growing at earlier stages of development, invested fewer resources on the development of the panicle and seed biomass. It should be noted that, for these plants, lower yields were not correlated with reduced seed weights, but with smaller panicles producing fewer seeds. This negative relation between plant height and seed yield and positive relation between panicle size (biomass and height) and yield (Table 3, Supplementary Figure 3) was previously reported in quinoa [32-34]. Furthermore, Gómez et al. [35] pointed the correlation between plant height and yield in quinoa and postulated that quinoa's low yield can be explained by its low sink capacity, and that an increase in reproductive partitioning, reducing plant height, could positively impact yield in this crop, as had happened previously to wheat and rice during the Green Revolution [36]. Also, some reports have found a positive correlation between yield and plant height, pointing as well to the influence of the environment in controlling this trait. Therefore, the implication of the environment should be investigated for the varieties here analyzed [37]. Nonetheless, plant height can be an important trait for breeding and further research analyzing endogenous factors that may control quinoa height and its relationship with yield and lodging (i.e. phytohormones) should be considered in quinoa.

Yield was highly determined by different physiological traits including panicle size and plant biomass, but only correlated with two seed traits: the germination capacity and the phenolic content (Supplementary Figure 3). Intriguingly, previous works have observed correlations between yield and seed nutritional-related traits, like the positive correlation found between yield and the antioxidant capacity or the K content, or the negative correlation between yield and protein content or the amount of different amino acids $[21,25,38]$. However, these studies compared the nutritional profiles of quinoa seeds harvested from different cultivars but grown in different environmental conditions (with variations in the sowing date, the cultivation location, and/or the year of cultivation), and the variations on the nutritional traits of the seeds were mainly determined by differences in the environmental conditions at seed filling stage, which affect both, yield and seed-quality traits [25]. In the present study, only the genetic factor was evaluated, so the lack of correlations between yield and seed nutritional-related traits suggests that there might be no link between them, and those relations are only relevant when introducing the cultivars to new environments where they are not yet adapted.

Downey mildew is one of the main diseases affecting quinoa on a global scale and it is caused by the fungus Peronospora variabilis Gäum [39]. Optimal conditions for mildew development are found at high humidity $(>80 \% \mathrm{RH})$ and moderate temperatures (between $18^{\circ} \mathrm{C}$ and $22^{\circ} \mathrm{C}$ ), but its expansion can be interrupted by long periods of sunny and dry conditions [39]. In this study, high RH was found in March, when most of the precipitations occurred and plants were still emerging or developing their first true leaves (Supplementary Figure 1). However, temperatures at that time were lower than $18^{\circ} \mathrm{C}$ so they were under the optimum for mildew development. Mildew produces chlorotic patches on leaves, which may result in premature defoliation by the plant as a defense mechanism. This reduction of the photosynthetic area can lead to an atrophied development and smaller panicles, which in turn lowers seed yield [40]. When the infection occurs at early stages of development of quinoa, $20 \%-40 \%$ yield penalties have been estimated for mildew-resistant cultivars [41], and losses of up to $99 \%$ in susceptible cultivars [40]. However, the impact of mildew on well established mature plants is less important than abiotic stresses [42], since the defoliation caused by the disease and by the natural senescence of the plant overlap [39]. In the present study, mildew incidence and severity were limited at early stages (82 and 100 d. a. s.), with severities lower than 10\% in most cultivars (Figure 3), but severity increased at a later stage (128 d. a. s.), being A-SE-02 cv. (cluster 2), A-SE09 cv., A-SE-10 cv., A-SE-11 cv., and A-SE-13 cv. (cluster 3) the most affected ones, and ASE-01 cv., A-SE-12 cv. (cluster 2), A-SE-03 cv., A-SE-06 (cluster 1), A-SE-04 cv., and A-SE$08 \mathrm{cv}$. (cluster 3) the least affected (Figure 3). Mildew severity did not correlate to yield (Supplementary Figure 3) nor to any seed nutritional traits (Supplementary Figure 3) [42], 
which suggests that the cultivars tested were resistant to mildew and did not suffer significant yield losses related to this disease despite not using plant protectants.

Saponins are considered as 'anti-nutrients' because of their negative effect on the bioavailability of minerals like Fe and Zn [43], together with the associated bitterness when they are present in substantial amounts in the seeds. Different breeding programs have been focused on the development of cultivars with very low seed saponin contents (sweet quinoa cultivars) [26]. Koziol [44] established the limit between sweet seed and bitter seed at $0.11 \%$ of seed weight, while Mastebroek et al. [45] considered sweet seeds those with saponin contents between $0.02 \%$ and $0.04 \%$ and bitter seeds those with contents between $0.47 \%$ and $1.13 \%$. All of our samples fit the definition of bitter seed by Koziol [44], but only A-SE-04, A-SE-08, A-SE-10 cv. (cluster 3), A-SE-12 cv., and A-SE-15 cv. (cluster 2) would be considered bitter following the Mastebroek et al. [45] criterion, and all samples would be 'low-saponin' seeds according to Medina-Meza et al. [28]. In this regard, it should be pointed that sweet varieties are normally preferred since the elimination process of saponins is avoided. However, some farmers prefer bitter cultivars because saponins may confer resistance to biotic stresses $[19,37]$ Although saponins have been hypothesized to also give mildew resistance to quinoa [47] since they possess antifungal activities [48], no correlation has been found between seed saponin contents and mildew resistance [49] (Supplementary Figure 3).

Saponin is a highly genotype-dependent seed trait in quinoa [50], and no correlation to other seed traits had been found previously. In the present study, we found, for the first time to our knowledge, a correlation between saponin content with other seed qualityrelated traits like germination, protein content, and flavonoid content (Supplementary Figure 3, [42,43,44].

Germination capacity is an important seed characteristic since any genetic potential achieved through breeding efforts cannot be exploited if seed establishment in the field is not successful. In this study, most cultivars surpassed the $50 \%$ germination rate, but ASE-13, A-SE-07, A-SE-01, and especially A-SE-03 and A-SE-06 (cluster 1), showed very low germination rates (Figures 5 and 9). Interestingly, a correlation between seed germination rates and the panicle's characteristics and seed yield of the mother plants was found [25] (Supplementary Figure 3), but germination rates were also influenced by nutritional traits of seeds. Both, the correlation and pathway analyses, showed a strong effect of the phenolic compounds and the P contents on the germination capacity of seeds (Supplementary Figure 3, Table 1). These results are in part supported by previous works, as it was already described a positive correlation between phenolic compounds and the germination capacity of quinoa seeds [25]. Furthermore, a stimulating effect of these compounds on the germination capacity has been reported as well in the close quinoa relative species Chenopodium album L. [54]. On the other hand, P is present in quinoa seeds mainly as phytate [55], a form of $\mathrm{P}$ storage not bioavailable for many monogastric animals, including humans $[47,48]$. During germination, however, the phytase activity catalyzes the hydrolysis of the phytate [58], providing inorganic phosphate essential for the metabolism of the seed at the beginning of germination [59,60]. According to Nadeem et al., [61], a higher phytate content also means more hydrolysis and thus, higher phosphate available during germination, which may explain the correlation between P content and germination (Table 1, Supplementary Figure 3 ), since higher P contents in seeds are related to faster germination and better establishment of seeds in the field [62]. A strong positive correlation was also found between the germination capacity and protein content (Supplementary Figure 3), probably associated with the role that storage proteins play on germination [63]. Noteworthy, it is expected that the environmental conditions may impact differently the cultivars included in this study resulting, consequently, in variations in seed germination [25, 65]. To explore further this aspect, the cultivation in consecutive years should be considered in future works.

In the present study, the protein content of seeds varied depending on the cultivar, with values ranging between $12.7 \%$ and $16.7 \%$ with the exception of two cultivars, the 
low-performing A-SE-03 and A-SE-06 (cluster 1). These two cultivars presented seed protein contents of $9 \%-10 \%$, closer to the values found in cereals like maize and barley and lower than the values found in wheat [65]. The contents of the rest of cultivars fell within the range expected for quinoa seeds, with A-SE-02, A-SE-12, A-SE-15 (cluster 2), and ASE-08 (cluster 3) exceeding 15\% [65]. However, it should be noted that the importance of the quinoa protein does not only rely on the quantity, but also on the quality, since it contains all amino acids which besides, are present in a proper balance, similar to the complete amino acid profile found in the cow milk and close to the ideal equilibrium recommended by the FAO for human consumption [66] [67].

The ash content ranged from $3.03 \%$ to $3.73 \%$ depending on the cultivar, although few significant differences were found among cultivars (Table 1) which were normal values for quinoa, but generally higher than those of cereals like wheat or rice $[65,66]$. The minerals that were present in higher amounts were $\mathrm{K}, \mathrm{P}, \mathrm{Ca}$, and $\mathrm{Mg}$, while $\mathrm{Na}, \mathrm{Fe}, \mathrm{Zn}, \mathrm{Mn}$, and $\mathrm{Cu}$ contents were the lowest (Table 1) [66]. All these minerals fell within the ranges previously reported for quinoa seeds [25,65], and some of them, like $\mathrm{K}, \mathrm{Ca}, \mathrm{Mg}$, and $\mathrm{Na}$, were higher than those found in cereals like maize, barley, rice, and wheat [67]. The high contents of $\mathrm{Fe}, \mathrm{Ca}$, and $\mathrm{Mg}$ are especially important since they are minerals less present in gluten-free products, and thus, quinoa seeds are an important source of these minerals for people with coeliac disease [66].

Noteworthy, the contents were significatively different among cultivars for all minerals except for $\mathrm{Mg}$ (Table 1). The variations in mineral contents in quinoa seeds had been previously reported to be cultivar-dependent, but they also respond to environmental differences during plant growth [21,25]. For instance, the cultivar A-SE-03 showed high Ca and $\mathrm{Na}$, and low $\mathrm{P}$ and $\mathrm{Zn}$ contents, while A-SE15 cv. showed high P, Cu, and Zn contents and low $\mathrm{Ca}, \mathrm{Na}$, and Fe contents (Table 1). P content was high, but according to Konishi et al. [55], $\mathrm{P}$ is mostly found in quinoa seeds as phytic acid, which can form complexes with $\mathrm{Fe}, \mathrm{Zn}, \mathrm{Mg}$, and $\mathrm{Ca}$, reducing their bioavailability for human digestion [68]. Interestingly, [43] pointed out that, in fed experimental experiments with rats, there were no differences in Fe availability in quinoa supplemented diets compared to those supplemented with $\mathrm{FeSO}_{4}$. Thus, further evaluation of the actual effect of quinoa seeds' phytic acid in $\mathrm{Fe}$, $\mathrm{Zn}, \mathrm{Mg}$, and Ca availability should be carried out in order to elucidate which percentage of these minerals' contents is actually taken up during human digestion and if these contents reach the human nutritional requirements [69].

A correlation between phenolic compounds and flavonoids contents and the antioxidant capacity was expected (Supplementary Figure 3) [25,70]. However, other compounds may have an antioxidant role on quinoa seeds, such as ascorbic acid, phytic acid, sterols, carotenoids, saponins, and even some proteins, may also have radical scavenging potential $[71,72]$. The antioxidant capacity and phenolic compounds content are genotypedependent in quinoa seeds [73], although they can also change depending on the environmental context $[20,25,74]$. In the present study, the antioxidant capacity, TPC, and TFC were comparable to those found in previous studies (Fig. 7) $[15,20,25,73]$ and changed depending on the cultivar. For instance, the cultivars A-SE-04 and A-SE-15, and A-SE-04, A-SE-08, and A-SE-10 showed the highest levels of antioxidant capacity and TPC, respectively, while A-SE-01 presented the lowest antioxidant capacity and A-SE-03 and A-SE-06 the lowest TPC and TFC (Figure 7). These results correlated well with other seed-related traits like protein content and germination rates, and with seed yield (Supplementary Figure 3). This, together with the overall health benefits of antioxidants, make of TPC and TFC interesting traits for quinoa breeding programs.

Targeting phenotypic traits such as physiological or seed nutritional-related parameters might be very useful when aiming at performing phenotyping screenings or for breeding programs. The selection of the best quinoa cultivars for production can be based on the results obtained for some of the parameters here discussed (including the results of their correlation). For instance, the present work highlights that the cultivar A-SE-08 is 
the most promising one amongst the 15 cultivars studied based on the higher protein contents, yield, germination rate, and $\mathrm{P}$ and phenolic contents. Targeting these traits can be very useful for selecting the best adapted varieties for a particular area of cultivation.

\section{Materials and Methods}

\subsection{Plant material, experimental design, and location}

Field trials were carried out in a field experimental station located in Lebrija (Seville, Spain, $36.88^{\circ} \mathrm{N}, 6.13^{\circ} \mathrm{W}$ ) in a clay-loam soil. Planting to harvesting dates took place from January to August of 2018. Fifteen different quinoa cultivars were used in this study, encoded as follows: 'A-SE-01', 'A-SE-02', 'A-SE-03', 'A-SE-04', 'A-SE-05', 'A-SE-06', 'A-SE07', 'A-SE-08', 'A-SE-09', 'A-SE-10', 'A-SE-11', 'A-SE-12', 'A-SE-13', 'A-SE-14', and 'A-SE$15^{\prime}$ and given by Algosur S.A. (Lebrija, Spain) (Supplementary Figure 4).

Each cultivar was sown on January 27th in two replicates of non-randomized plots, with dimensions of $4.5 \mathrm{~m} \times 266 \mathrm{~m}$, spacing between rows of $0.75 \mathrm{~m}$ and $0.02 \mathrm{~m}$ within rows. A drilling machine was used to sow with a density of seeds of 2 kg.ha-1.

During the experiment, different measurements of physiological traits were taken. Plant height and downy mildew incidence and severity were measured at 82, 100, and 128 days after sowing (d.a.s), which corresponded to different developmental stages: fully emerged plants, panicle emergence, and beginning of seed ripening, respectively. Also, at 128 d.a.s., panicle length and weight of 25 plants per cultivar were measured. Plant harvesting took place when plants had naturally dried out at different time points: July 18th (172 days after sowing) for the cultivars 'A-SE-03' cv. 'A-SE-06' cv. , 'A-SE-07' cv., 'A-SE-09' cv., 'ASE-12' cv., 'A-SE-13' cv., and 'A-SE-15' cv., and in August 1st (186 d.a.s.) for 'A-SE-01' cv., 'A-SE-02' cv., 'A-SE-04' cv., 'A-SE-05' cv., 'A-SE-08' cv., 'A-SE-10' cv., 'A-SE-11' cv., and 'A-SE-14' cv. Total seed yield was quantified from an $11.25 \mathrm{~m}^{2}$ plot for each cultivar and the dry weight of 20 plants was measured.

Climatological data, including total precipitation, relative humidity (RH), and temperature was obtained daily from a local climatological station (Supplementary Figure 1). Sprinkler irrigation was supplemented at different developmental stages: at seed sowing $\left(30 \mathrm{l} / \mathrm{m}^{2}\right), 5$ days after sowing $\left(30 \mathrm{l} / \mathrm{m}^{2}\right)$, at the beginning of branching $\left(30 \mathrm{l} / \mathrm{m}^{2}\right)$, at flowering $\left(50 \mathrm{l} / \mathrm{m}^{2}\right)$, and during grain filling $\left(50 \mathrm{l} / \mathrm{m}^{2}\right)$.

\subsection{Seed weight and area.}

Seeds were manually counted and weighed in an analytical balance. Seed area was analyzed using the open-source software ImageJ (http://rsbweb.nih.gov/ij/). Images were taken using an Olympus SZ61 stereomicroscope (Olympus Corporation, Shinjuku, Tokyo, Japan) and processed with the AnalySIS GetIT image software (analysis getIT 5.1, Olympus Corporation, Shinjuku, Tokyo, Japan). To determine seed weight, 1000 seeds were used per replication and 3 replications were utilized. For seed area measurement, 50 seeds were used per replication and 3 replications were utilized

\subsection{Seed germination rate}

Quinoa seeds (using 50 seeds per replication and 3 replications per cultivar) were sterilized by soaking first in ethanol $70 \%$ for two minutes, next in bleach $50 \%$ with a droplet of Tween-20 for two minutes, and then rinsing several times in distilled water $\left(\mathrm{H}_{2} \mathrm{O}\right)$. Sterilized seeds were sown on a double layer of filter paper wet with distilled water on Petri dishes and then transferred to a growth chamber under darkness and a controlled temperature of $23^{\circ} \mathrm{C}$. Germination rate was counted daily for the first week after sowing. Seeds were considered as germinated when the radicle protrusion was longer than $2 \mathrm{~mm}$. 


\subsection{Seed viability}

Seed viability tests were performed using the tetrazolium method (2,3,5-triphenyl-2H-tetrazolium chloride). First, seeds (using 100 seeds per replication and 3 replications per cultivar) were imbibed in distilled water at $30^{\circ} \mathrm{C}$ for an hour to facilitate longitudinal and superficial cuts of the embryo and to ensure a homogeneous dying of the seed tissues. After cutting, seeds were submerged in $1 \%$ tetrazolium chloride at $30^{\circ} \mathrm{C}$ for two hours. Seeds with more than $50 \%$ staining in the embryonic tissue were considered viable.

\subsection{Saponin content}

To determine saponin content, $20 \mathrm{~mL}$ of $50 \%$ ethanol was added to $1 \mathrm{~g}$ of powdered sample and left to macerate for $72 \mathrm{~h}$ at room temperature. Then, the extracts were filtered into $20 \mathrm{~mL}$ volumetric flasks. The samples were then filtered using a $0.45 \mu \mathrm{m}$ nylon Filter-Lab syringe and analyzed by High-Performance Liquid Chromatography Fluorescence and Diodo Array Detection (HPLCDAD, Serie 1100, Agilent Technologies, Waldbronn, Germany) at $225 \mathrm{~nm}$ [75]. Saponin (Merck, Germany) was used as a standard. The results were expressed in g saponin $100 \mathrm{~g}^{-1}$ of fresh weight.

\subsection{Protein content}

The protein content was determined according to AOAC Official Methods [76], using an elemental analyzer Leco TruSpec (LECO TruSpec (LECO, Michigan, USA)) and considering a conversion factor of 6.25 [77].

\subsection{Mineral content}

The mineral content was analyzed following the official methods of analysis of the Spanish Ministry of Agriculture (MAPA, 1995). Phosphorus content was determined using a spectrophotometer UV-VIS (Hitachi U-2810, Tokyo, Japan) (yellow coloration, $430 \mathrm{~nm}$ ). Potassium was determined through flame atomic emission spectroscopy. Calcium, magnesium, sodium, iron, copper, manganese, and zinc contents were assessed using flame atomic absorption spectroscopy (AAS) (SpectrAA 110, Agilent Technologies Inc., Palo Alto, CA, USA) after mineralizing the samples with $\mathrm{H}_{2} \mathrm{O}$ and $\mathrm{HCl}(35 \%)$.

\subsection{Ferric reducing antioxidant power (FRAP) assay.}

To obtain total extracts, seeds were ground to a fine powder and $100 \mathrm{mg}$ of the flour were homogenized in $1 \mathrm{~mL}$ of an extraction buffer consisting of methanol (50\%), acetic acid $(1 \%)$, and distilled water $(49 \%)$. These samples were vortexed for 2 minutes and kept in the dark at $4^{\circ} \mathrm{C}$ for 48 hours, before centrifugation for 15 minutes at $13500 \mathrm{rpm}$. The supernatants were stored at $-20^{\circ} \mathrm{C}$ until their use in the FRAP and flavonoid content assays.

The antioxidant capacity of seeds was determined following the procedure described by Benzie and Strain [78]. The FRAP reagent consisted of a mix of $300 \mathrm{mM}$ acetate buffer ( $\mathrm{pH}$ 3.6), with $10 \mathrm{mM}$ TPTZ in $40 \mathrm{mM} \mathrm{HCl}$ and $20 \mathrm{mM} \mathrm{FeCl}_{3} \cdot 6 \mathrm{H}_{2} \mathrm{O}$ at a ratio of 10:1:1 (v/v/v). Twenty $\mu \mathrm{L}$ of sample extract and $180 \mu \mathrm{L}$ of FRAP reagent were added into a 96-well microplate and, after 4 minutes, absorbance was read at $593 \mathrm{~nm}$ using a microplate reader Lector Multi-ModalSynergy HTX (BioTek Instruments, Inc., USA). The antioxidant capacity was calculated from a calibration curve obtained with iron (II) sulfate $\left(\mathrm{FeSO}_{4}\right)$. FRAP value was expressed as $\mu \mathrm{mol}$ of $\mathrm{Fe}^{2+} \mathrm{g}^{-1}$ of seed. 
Extracts were obtained homogenizing $100 \mathrm{mg}$ seed flour in $1 \mathrm{~mL}$ of ice-cold methanol (95\%), vortexing, and centrifuging at $13500 \mathrm{rpm}$ for 5 minutes after $48 \mathrm{~h}$ kept in the dark and at $4^{\circ} \mathrm{C}$.

The content of polyphenols was measured following the protocol described by Ainsworth and Gillespie [79]. Briefly, the mixture of $100 \mu \mathrm{L}$ of sample extract or standard and $200 \mu \mathrm{L}$ of the Folin-Ciocalteu reagent $10 \%$ was vortexed for 1 minute before adding $800 \mu \mathrm{L}$ of sodium carbonate $7.5 \%$ and was then incubated for 2 hours in the dark. The samples were centrifuged in order to eliminate precipitates. Absorbance was read at $765 \mathrm{~nm}$ using a microplate reader Lector Multi-ModalSynergy HTX (BioTek Instruments, Inc., USA). Concentrations of gallic acid between $20 \mu \mathrm{g} \cdot \mathrm{mL}^{-1}$ and $200 \mu \mathrm{g} \cdot \mathrm{mL}^{-1}$ in methanol (95\%) were used as standard, and thus the TPC was expressed as mg of gallic acid equivalents per grams of quinoa seed (mg GAE.g-1).

\subsection{Total flavonoid content (TFC)}

Flavonoid content was determined following the procedure described by Valenzuela [80]. The same extracts as in the FRAP assay were used. Briefly, $30 \mu \mathrm{L}$ of sample extract or standard, $10 \mu \mathrm{L}$ of aluminum chloride $\left(\mathrm{AlCl}_{3}\right) 10 \%, 10 \mu \mathrm{L}$ of sodium acetate $\left(\mathrm{NaC}_{2} \mathrm{H}_{3} \mathrm{O}_{2}\right)$ $1 \mathrm{M}$, and $250 \mu \mathrm{L}$ of $\mathrm{dH} 2 \mathrm{O}$ were mixed and incubated for 30 minutes. The absorbance was read at $415 \mathrm{~nm}$ using a microplate reader Lector Multi-ModalSynergy HTX (BioTek Instruments, Inc., USA). Quercetin dissolved in ethanol (80\%) was used as standard with concentrations ranging from $10 \mu \mathrm{g} \cdot \mathrm{mL}^{-1}$ to $140 \mu \mathrm{g} \cdot \mathrm{mL}^{-1}$. The results were expressed in mg of quercetin equivalents per gram of quinoa seed (mg QE. $\left.\mathrm{g}^{-1}\right)$.

\subsection{Statistical analysis}

To analyze the differences between cultivars different one-way ANOVA tests were performed. For variables where normality and equal variances could be assumed, a One-way ANOVA test was performed, followed by a Tukey post-hoc test, to perform multiple comparisons at a probability level of $5 \%(\mathrm{p}<0.05)$. A one-way ANOVA on ranks (KrustalWallis test by ranks) was performed when data did not present a normal distribution, and a Welch's ANOVA test followed by a Games-Howell post-hoc test was performed when variances were not equal, both at a probability level of $5 \%(p<0.05)$. Normality and equality of variances of the data were tested through a Kolmogorov-Smirnov's test and a Levene's, respectively. A sequential path analysis was performed to evaluate the specific contribution of different physiological traits to yield. This analysis allows ordering different variables as predictors of yield of first, second, or third-order [81]. For this purpose, a stepwise multiple linear regression procedure was used where variables that showed weak contribution $(p>0.05)$ to the dependent variable (yield) or high multicollinearity, were automatically dropped from the model. The variables entered into the model were considered as first-order predictors and the procedure was repeated using these variables as the response variable to identify traits that function as second-order predictors of yield. Tolerance and variance inflation factor (VIF) were used to measure the level of multicollinearity for each predictor trait, considering tolerance lower than 0.1 or VIF values higher than 10 as high levels of collinearity. Tolerance (1- R2i, where R2i is the coefficient of determination for the prediction of variable $i$ by the predictor variables) is the amount of variance of the selected independent variable not explained by other independent variables. VIF (1/Tolerance) indicates the extent of the effects of other independent variables on the variability of the selected independent variable. Principal component analysis was performed for plant parameters, like plant height at three stages, panicle length and biomass, plant biomass, mildew severity at different stages, resistance to lodging, life-cycle length and yield, and for seed parameters, like viability and germination rates, 1000 seeds' weight, seed area, saponin content, $\mathrm{N}$ and protein content and C-N ratio, FRAP value, phenols and flavonoids contents, and mineral contents. Correlations amongst variables 
were evaluated with a Pearson's correlation coefficient test. The SPSS Statistics 23.0 (SPSS Inc.) package was used for the statistical analyses.

\section{Conclusions}

Overall, this study reveals differences among cultivars for each physiological and seed nutritional-related trait analyzed, although there were similarities among some cultivars. For instance, A-SE-03 and A-SE-06, cluster together in the PCA, showed taller plants at early stages of development but shorter plants with smaller panicles and lower yields at maturity (Figures 1 and 9, Supplementary Figure 3). Regarding seed traits, they presented lower germination rates, and lower protein, $\mathrm{P}$, phenols, flavonoids, and saponins contents (Figures 5, 7, 8, and 9, Table 1). The most promising cultivars for this agroclimatic context are those included in cluster 3 , due to the higher yields, germination rates, and TPC (Figure 9). Yield is already the most important selection trait in breeding programs, since higher yields ensure higher productivity [26]. To keep an adequate germination rate is also an important agronomical trait in quinoa [82]. Also, TPC can be an interesting trait to explore in breeding programs, since polyphenols are bioactive compounds that can improve shelf-life of the product and provide health benefits to the consumers [12]. Quinoa seeds are well known to be an excellent source of high-quality protein of nonanimal origin. This is one of the main traits that makes quinoa a crop with a high nutritional quality, important for achieving food security locally and globally [83]. In line with this, also protein contents are relevant from a nutritional point of view. In this study, the higher protein contents were shown by A-SE- 15 cv., A-SE-02 cv., A-SE-12 cv., and A-SE$08 \mathrm{cv}$. Thus, the cultivar A-SE-08 (cluster 3) not only presented a high protein content, but also higher yield, germination rate, and $\mathrm{P}$ and phenolic contents, all of these making this cultivar one of the most promising ones for this particular agronomical area. Having all these traits positively correlated can greatly facilitate the development of a better adapted cultivar. However, it should be noted that saponin content was also higher in this cultivar (Figure 9). Saponin content is one of the main targets in breeding programs, as reducing the saponin content can improve nutritional quality and flavor $[19,68]$. Saponins are located in the external coat of the episperm, so they can be partially removed from seeds by using abrasive methods and/or washing with cold water [85]. However, these processes can be costly and time-consuming [86]. Pulvento et al. [87] and Gómez-Caravaca et al. [53] have reported that agronomical management practices can lower the saponin content so it would be interesting to study these aspects when establishing this crop cultivation.

Therefore, the results here presented highlight the importance of considering the genotypic variation in quinoa when selecting improved quinoa varieties with better nutritional characteristics for new cultivation environments. Further studies are required to determine which exact parameters are genotype-variable and which ones show genotypic stability.

Supplementary Materials: The following are available online at www.mdpi.com/xxx/s1, Figure S1: title, Table S1: title, Video S1: title.

Author Contributions: M.R., J.M., and S.V. conceived and planned the experiments. S.G.R., J.M., S.V., I.M.G., J.M.P., M.J.R., and P.C. carried out the experiments. M.R., S.V., S.G.R., I.M., J.M., M.R., V.C., and L.B. contributed to the interpretation of the results. M.R., I.M.G, and S.G.R. took the lead in writing the manuscript. All authors provided critical feedback and helped shape the research, analysis, and manuscript.

Funding: This work was supported by the Ministerio de Ciencia e Innovación (MICINN, Spain) (PID2019-105748RA-I00), the Madrid Government (Comunidad de Madrid-Spain) under the Multiannual Agreement with Universidad Autónoma de Madrid in the line of action encouraging youth research doctors, in the context of the V PRICIT (Regional Programme of Research and Technological Innovation) (SI1/PJI/2019-00124), the CYTED (ValSe-Food 119RT0567), the FPI UAM Fellowship Programme 2019 (to SG-R), the CM research assistant fellowship (to I. M. G.) and the Ramón y Cajal Programme 2019 (to MR). 
Data Availability Statement: The data that support the findings of this study are available upon request.

Acknowledgments: The authors greatly thank ALGOSUR S.A for providing the quinoa seeds used in this study.

Conflicts of Interest: The authors declare no conflict of interest

\section{References}

1. Zou, C.; Chen, A.; Xiao, L.; Muller, H.M.; Ache, P.; Haberer, G.; Zhang, M.; Jia, W.; Deng, P.; Huang, R.; et al. A high-quality genome assembly of quinoa provides insights into the molecular basis of salt bladder-based salinity tolerance and the exceptional nutritional value. Cell Res. 2017, 27, 1327-1340, doi:10.1038/cr.2017.124.

2. Ruiz, K.B.; Biondi, S.; Oses, R.; Acuña-rodríguez, I.S.; Antognoni, F.; Martinez-Mosqueira, E.A.; Coulibaly, A.; CanahuaMurillo, A.; Pinto, M.; Zurita-Silva, A.; et al. Quinoa biodiversity and sustainability for food security under climate change . A review. Agron. Sustain. Dev. 2014, 34, 349-359, doi:10.1007/s13593-013-0195-0.

3. Wu, Q.; Bai, X.; Wu, X.; Xiang, D.; Wan, Y.; Luo, Y.; Shi, X.; Li, Q.; Zhao, J.; Qin, P.; et al. Transcriptome profiling identifies transcription factors and key homologs involved in seed dormancy and germination regulation of Chenopodium quinoa. Plant Physiol. Biochem. 2020, 151, 443-456, doi:10.1016/j.plaphy.2020.03.050.

4. Alandia, G.; Rodriguez, J.P.; Jacobsen, S.-E.; Bazile, D.; Condori, B. Global expansion of quinoa and challenges for the Andean region. Glob. Food Sec. 2020, 26, 100429, doi:10.1016/j.gfs.2020.100429.

5. Bazile, D.; Jacobsen, S.E.; Verniau, A. The Global Expansion of Quinoa : Trends and Limits. Front. Plant Sci. 2016, 7, 622, doi:10.3389/fpls.2016.00622.

6. Hernandez, R.; Cardoso F, Q.; Diaz, C.; Salazar, C.; Morales, A.; Lagunas B, C.; Guillen, J.; Mancera, V.; Salem, A.Z.M.; Pérez, O.; et al. (2s) Acacia farnesiana and Acacia cochliacantha. Life Sci. J. 2015, 12, 1097-8135.

7. Repo-Carrasco, R.; Espinoza, C.; Jacobsen, S.E. Nutritional value and use of the andean crops quinoa (Chenopodium quinoa) and kañiwa (Chenopodium pallidicaule). Food Rev. Int. 2003, 19, 179-189, doi:10.1081/FRI-120018884.

8. Jarvis, D.E.; Ho, Y.S.; Lightfoot, D.J.; Schmöckel, S.M.; Li, B.; Borm, T.J.A.; Ohyanagi, H.; Mineta, K.; Michell, C.T.; Saber, N.; et al. The genome of Chenopodium quinoa. 2017, doi:10.1038/nature21370.

9. Liu, Y.; Kong, Z.; Liu, J.; Zhang, P.; Wang, Q.; Huan, X.; Li, L.; Qin, P. Non-targeted metabolomics of quinoa seed filling period based on liquid chromatography-mass spectrometry. Food Res. Int. 2020, 137, doi:10.1016/j.foodres.2020.109743.

10. Asher, A.; Galili, S.; Whitney, T.; Rubinovich, L. The potential of quinoa (Chenopodium quinoa) cultivation in Israel as a dual-purpose crop for grain production and livestock feed. Sci. Hortic. (Amsterdam). 2020, 272, 109534, doi:10.1016/j.scienta.2020.109534.

11. Rodríguez Gómez, M.J.; Matías Prieto, J.; Cruz Sobrado, V.; Calvo Magro, P. Nutritional characterization of six quinoa (Chenopodium quinoa Willd) varieties cultivated in Southern Europe. J. Food Compos. Anal. 2021, 99, 1-7, doi:10.1016/j.jfca.2021.103876.

12. Tang, Y.; Tsao, R. Phytochemicals in quinoa and amaranth grains and their antioxidant, anti-inflammatory, and potential health beneficial effects: a review. Mol. Nutr. Food Res. 2017, 61, 1600767, doi:10.1002/mnfr.201600767.

13. Vega-Gálvez, A.; Miranda, M.; Vergara, J.; Uribe, E.; Puente, L.; Martínez, E.A. Nutrition facts and functional potential of quinoa (Chenopodium quinoa willd.), an ancient Andean grain: A review. J. Sci. Food Agric. 2010, 90, 2541-2547, doi:10.1002/jsfa.4158.

14. Benavente-García, O.; Castillo, J. Update on uses and properties of citrus flavonoids: New findings in anticancer, cardiovascular, and anti-inflammatory activity. J. Agric. Food Chem. 2008, 56, 6185-6205, doi:10.1021/jf8006568.

15. Park, J.H.; Lee, Y.J.; Kim, Y.H.; Yoon, K.S. Antioxidant and antimicrobial activities of Quinoa (Chenopodium quinoa Willd.) Seeds Cultivated in Korea. Prev. Nutr. Food Sci. 2017, 22, 195-202, doi:10.3746/pnf.2017.22.3.195.

16. Gorinstein, S.; Vargas, O.J.M.; Jaramillo, N.O.; Salas, I.A.; Ayala, A.L.M.; Arancibia-Avila, P.; Toledo, F.; Katrich, E.; 
Trakhtenberg, S. The total polyphenols and the antioxidant potentials of some selected cereals and pseudocereals. Eur. Food Res. Technol. 2007, 225, 321-328, doi:10.1007/s00217-006-0417-7.

17. Muziri, T.; Chaibva, P.; Chofamba, A.; Madanzi, T.; Mangeru, P.; Mudada, N.; Manhokwe, S.; Mugari, A.; Matsvange, D.; Murewi, C.T.F.; et al. Using principal component analysis to explore consumers' perception toward quinoa health and nutritional claims in Gweru, Zimbabwe. Food Sci. Nutr. 2021, 9, 1025-1033, doi:10.1002/fsn3.2071.

18. Cramer, W.; Guiot, J.; Fader, M.; Garrabou, J.; Gattuso, J.-P.; Iglesias, A.; Lange, M.A.; Lionello, P.; Llasat, M.C.; Paz, S.; et al. Climate change and interconnected risks to sustainable development in the Mediterranean. Nat. Clim. Chang. 2018 811 2018, 8, 972-980, doi:10.1038/s41558-018-0299-2.

19. El estado mundial de la agricultura y la alimentación, 1994.

20. Aloisi, I.; Parrotta, L.; Ruiz, K.B.; Landi, C.; Bini, L.; Cai, G.; Biondi, S.; Duca, S. Del New Insight into Quinoa Seed Quality under Salinity: Changes in Proteomic and Amino Acid Profiles, Phenolic Content, and Antioxidant Activity of Protein Extracts. Front. Plant Sci. 2016, 7, 656, doi:10.3389/fpls.2016.00656.

21. Reguera, M.; Conesa, C.M.; Gil-Gómez, A.; Haros, C.M.; Pérez-Casas, M.Á.; Briones-Labarca, V.; Bolaños, L.; Bonilla, I.; Álvarez, R.; Pinto, K.; et al. The impact of different agroecological conditions on the nutritional composition of quinoa seeds. PeerJ 2018, 6:e4442, doi:10.7717/peerj.4442.

22. Gonzalez, J.A.; Konishi, Y.; Bruno, M.; Valoy, M.; Prado, F.E. Interrelationships among seed yield, total protein and amino acid composition of ten quinoa (Chenopodium quinoa) cultivars from two different agroecological regions. J. Sci. Food Agric. 2012, 92, 1222-1229, doi:10.1002/jsfa.4686.

23. Bertero, H.D.; De La Vega, A.J.; Correa, G.; Jacobsen, S.E.; Mujica, A. Genotype and genotype-by-environment interaction effects for grain yield and grain size of quinoa (Chenopodium quinoa Willd.) as revealed by pattern analysis of international multi-environment trials. F. Crop. Res. 2004, 89, 299-318, doi:10.1016/j.fcr.2004.02.006.

24. Matías, J.; Rodríguez, M.J.; Cruz, V.; Calvo, P.; Reguera, M. Heat stress lowers yields, alters nutrient uptake and changes seed quality in quinoa grown under Mediterranean field conditions. J. Agron. Crop Sci. 2021, 207, 481-491, doi:10.1111/jac.12495.

25. Granado-Rodríguez, S.; Aparicio, N.; Matías, J.; Pérez-Romero, L.F.; Maestro, I.; Garcés, I.; Pedroche, J.J.; Haros, C.M.; Fernández-García, N.; Navarro del Hierro, J.; et al. Studying the Impact of Different Field Environmental Conditions on Seed Quality of Quinoa: The Case of Three Different Years Changing Seed Nutritional Traits in Southern Europe. Front. Plant Sci. 2021, 12, 649132, doi:10.3389/fpls.2021.649132.

26. Zurita-Silva, A.; Fuentes, F.; Zamora, P.; Jacobsen, S.E.; Schwember, A.R. Breeding quinoa (Chenopodium quinoa Willd.): Potential and perspectives. Mol. Breed. 2014, 34, 13-30, doi:10.1007/s11032-014-0023-5.

27. Koziol, M.J. Afrosimetric estimation of threshold saponin concentration for bitterness in quinoa (Chenopodium quinoa Willd). J. Sci. Food Agric. 1991, 54, 211-219, doi:10.1002/jsfa.2740540206.

28. Medina-Meza, I.G.; Aluwi, N.A.; Saunders, S.R.; Ganjyal, G.M. GC-MS Profiling of Triterpenoid Saponins from 28 Quinoa Varieties (Chenopodium quinoa Willd.) Grown in Washington State. J. Agric. Food Chem. 2016, 64, 8583-8591, doi:10.1021/acs.jafc.6b02156.

29. Alencar, N.M.M.; Carvalho Oliveira, L. de Advances in Pseudocereals: Crop Cultivation, Food Application, and Consumer Perception. Ref. Ser. Phytochem. 2019, 1695-1713, doi:10.1007/978-3-319-78030-6_63.

30. Abugoch James, L.E. Quinoa (Chenopodium quinoa Willd.): Composition, Chemistry, Nutritional, and Functional Properties. In Advances in Food and Nutrition Research; Elsevier Inc., 2009; Vol. 58, pp. 1-31.

31. Fuentes, F.; Bhargava, A. Morphological Analysis of Quinoa Germplasm Grown Under Lowland Desert Conditions. J. Agron. Crop Sci. 2011, 197, 124-134, doi:10.1111/J.1439-037X.2010.00445.X.

32. Miranda, M.; Vega-Gálvez, A.; Martinez, E.; López, J.; Rodríguez, M.J.; Henríquez, K.; Fuentes, F. Genetic diversity and comparison of physicochemical and nutritional characteristics of six quinoa (Chenopodium quinoa willd.) genotypes cultivated in Chile. Food Sci. Technol. 2012, 32, 835-843, doi:10.1590/s0101-20612012005000114.

33. Benlhabib, O.; Boujartani, N.; Maughan, P.J.; Jacobsen, S.E.; Jellen, E.N. Elevated genetic diversity in an F2:6 population of 
quinoa (chenopodium quinoa) developed through an inter-ecotype cross. Front. Plant Sci. 2016, 7, 1-9, doi:10.3389/fpls.2016.01222.

34. Pulvento, C.; Riccardi, M.; Lavini, A.; D'Andria, R.; Iafelice, G.; Marconi, E. Field Trial Evaluation of Two Chenopodium quinoa Genotypes Grown Under Rain-Fed Conditions in a Typical Mediterranean Environment in South Italy. J. Agron. Crop Sci. 2010, 196, 407-411, doi:10.1111/j.1439-037X.2010.00431.x.

35. Gómez, M.B.; Castro, P.A.; Mignone, C.; Bertero, H.D. Can yield potential be increased by manipulation of reproductive partitioning in quinoa (Chenopodium quinoa)? Evidence from gibberellic acid synthesis inhibition using Paclobutrazol. Funct. Plant Biol. 2011, 38, 420-430, doi:10.1071/FP10168.

36. Sakamoto, T.; Matsuoka, M. Generating high-yielding varieties by genetic manipulation of plant architecture. Curr. Opin. Biotechnol. 2004, 15, 144-147, doi:10.1016/j.copbio.2004.02.003.

37. Maliro, M.F.A.; Guwela, V.F.; Nyaika, J.; Murphy, K.M. Preliminary Studies of the Performance of Quinoa (Chenopodium quinoa Willd.) Genotypes under Irrigated and Rainfed Conditions of Central Malawi. Front. Plant Sci. 2017, 8, doi:10.3389/FPLS.2017.00227.

38. Curti, R.N.; Sanahuja, M. del C.; Vidueiros, S.M.; Pallaro, A.N.; Bertero, H.D. Trade-off between seed yield components and seed composition traits in sea level quinoa in response to sowing dates. Cereal Chem. 2018, 95, 734-741, doi:10.1002/cche.10088.

39. Gandarillas, A.; Rojas, W.; Bonifacio, A.; Ojeda, N. La Quinua en Bolivia : perspectiva de la Fundación; 2015;

40. Danielsen, S.; Munk, L. Evaluation of disease assessment methods in quinoa for their ability to predict yield loss caused by downy mildew. Crop Prot. 2004, 23, 219-228, doi:10.1016/j.cropro.2003.08.010.

41. Danielsen, S.; Bonifacio, A.; Ames, T. Diseases of quinoa (Chenopodhim quinoa). Food Rev. Int. 2003, 19, 43-59, doi:10.1081/FRI-120018867.

42. Jacobsen, S.E. The scope for adaptation of quinoa in Northern Latitudes of Europe. J. Agron. Crop Sci. 2017, 203, 603-613, doi:10.1111/jac.12228.

43. Ruales, J.; Nair, B.M. Content of fat, vitamins and minerals in quinoa (Chenopodium quinoa, Willd) seeds. Food Chem. 1993, 48, 131-136, doi:10.1016/0308-8146(93)90047-J.

44. Wahli, C. Quinua: hacia su cultivo comercial; Wahli, C., Ed.; Latinreco S. A.: Quito, 1990; ISBN 9978990135.

45. Mastebroek, H.D.; Limburg, H.; Gilles, T.; Marvin, H.J.P. Occurrence of sapogenins in leaves and seeds of quinoa (Chenopodium quinoa Willd). J. Sci. Food Agric. 2000, 80, 152-156, doi:10.1002/(SICI)1097-0010(20000101)80:1<152::AIDJSFA503>3.0.CO;2-P.

46. Ricardo, S.M.; Karine, N.; Kurt, H. Novel molluscicide against Pomacea canaliculata based on quinoa (Chenopodium quinoa) saponins. Crop Prot. 2008, 27, 310-319, doi:10.1016/J.CROPRO.2007.03.015.

47. Khalifa, W.; Thabet, M. Variation in downy mildew ( Peronospora variabilis Gäum ) resistance of some quinoa ( Chenopodium quinoa Willd ) cultivars under Egyptian conditions. Middle East J. Agric. Res. 2018, 7, 671-682.

48. Woldemichael, G.M.; Wink, M. Identification and biological activities of triterpenoid saponins from Chenopodium quinoa. J. Agric. Food Chem. 2001, 49, 2327-2332, doi:10.1021/jf0013499.

49. Colque-Little, C.; Abondano, M.C.; Lund, O.S.; Amby, D.B.; Piepho, H.P.; Andreasen, C.; Schmöckel, S.; Schmid, K. Genetic variation for tolerance to the downy mildew pathogen Peronospora variabilis in genetic resources of quinoa (Chenopodium quinoa). BMC Plant Biol. 2021, 21, 1-19, doi:10.1186/s12870-020-02804-7.

50. Santis, G. De; Maddaluno, C.; D’Ambrosio, T.; Rascio, A.; Rinaldi, M.; Troisi, J. Characterisation of quinoa (Chenopodium quinoaWilld.) accessions for the saponin content in Mediterranean environment. Ital. J. Agron. 2016, 11, 277-281, doi:10.4081/ija.2016.774.

51. Escribano, J.; Cabanes, J.; Jiménez-Atiénzar, M.; Ibañez-Tremolada, M.; Gómez-Pando, L.R.; García-Carmona, F.; GandíaHerrero, F. Characterization of betalains, saponins and antioxidant power in differently colored quinoa (Chenopodium quinoa) varieties. Food Chem. 2017, 234, 285-294, doi:10.1016/j.foodchem.2017.04.187. 
(Chenopodium quinoa willd.) cultivars grown under field conditions in southwestern Germany. Agronomy 2018, 8, doi:10.3390/agronomy8100197.

53. Gómez-Caravaca, A.M.; Iafelice, G.; Lavini, A.; Pulvento, C.; Caboni, M.F.; Marconi, E. Phenolic compounds and saponins in quinoa samples (Chenopodium quinoa Willd.) grown under different saline and nonsaline irrigation regimens. J. Agric. Food Chem. 2012, 60, 4620-4627, doi:10.1021/jf3002125.

54. Reigosa, M.J.; Souto, X.C.; Gonz'lez, L. Effect of phenolic compounds on the germination of six weeds species. Plant Growth Regul. 1999282 1999, 28, 83-88, doi:10.1023/A:1006269716762.

55. Konishi, Y.; Hirano, S.; Tsuboi, H.; Wada, M. Distribution of minerals in quinoa (Chenopodium quinoa Willd.) seeds. Biosci. Biotechnol. Biochem. 2004, 68, 231-234, doi:10.1271/bbb.68.231.

56. Kumar, A.; Lingadurai, S.; Jain, A.; Barman, N. Erythrina variegata Linn: A review on morphology, phytochemistry, and pharmacological aspects. Pharmacogn. Rev. 2010, 4, 147-152, doi:10.4103/0973-7847.70908.

Holm, M.; Ma, L.G.; Qu, L.J.; Deng, X.W. Two interacting bZIP proteins are direct targets of COP1-mediated control of lightdependent gene expression in Arabidopsis. Genes Dev. 2002, 16, 1247-1259, doi:10.1101/gad.969702.

58. Honke, J.; Kozłowska, H.; Vidal-Valverde, C.; Frias, J.; Górecki, R. Changes in quantities of inositol phosphates during maturation and germination of legume seeds. Eur. Food Res. Technol. 1998, 206, 279-283.

59. Hall, J.R.; Hodges, T.K. Phosphorus Metabolism of Germinating Oat Seeds. Plant Physiol. 1966, 41, 1459-1464, doi:10.1104/pp.41.9.1459.

60. White, P.J.; Veneklaas, E.J. Nature and nurture: The importance of seed phosphorus content. Plant Soil 2012, 357, 1-8, doi:10.1007/s11104-012-1128-4.

61. Nadeem, M. UNIVERSITY OF BORDEAUX 1 Sciences and Environments Graduate School Remobilization of seed phosphorus reserves and exogenous phosphorus uptake during germination and early growth stages of maize (Zea mays L.). 2011.

62. Grant, C.A.; Flaten, D.N.; Tomasiewicz, D.J.; Sheppard, S.C. The importance of early season phosphorus nutrition. Can. J. Plant Sci. 2001, 81, 211-224, doi:10.4141/P00-093.

63. Koyro, H.W.; Eisa, S.S. Effect of salinity on composition, viability and germination of seeds of Chenopodium quinoa Willd. Plant Soil 2008, 302, 79-90, doi:10.1007/s11104-007-9457-4.

64. Pilco-Quesada, S.; Tian, Y.; Yang, B.; Repo-Carrasco-Valencia, R.; Suomela, J.P. Effects of germination and kilning on the phenolic compounds and nutritional properties of quinoa (Chenopodium quinoa) and kiwicha (Amaranthus caudatus). J. Cereal Sci. 2020, 94, 102996, doi:10.1016/j.jcs.2020.102996.

65. Koziol, M.J. Chemical Composition and Nutritional Evaluation of Quinoa (Chenopodium quinoa Willd.). J. Food Compos. Anal. 1992, 5, 35-68.

66. Filho, A.M.M.; Pirozi, M.R.; Borges, J.T.D.S.; Pinheiro Sant'Ana, H.M.; Chaves, J.B.P.; Coimbra, J.S.D.R. Quinoa: Nutritional, functional, and antinutritional aspects. Crit. Rev. Food Sci. Nutr. 2017, 57, 1618-1630, doi:10.1080/10408398.2014.1001811.

67. Repo-Carrasco, R.; Espinoza, C.; Jacobsen, S.E. Nutritional value and use of the andean crops quinoa (Chenopodium quinoa) and kañiwa (Chenopodium pallidicaule). Food Rev. Int. 2003, 19, 179-189, doi:10.1081/FRI-120018884.

68. Schlemmer, U.; Frølich, W.; Prieto, R.M.; Grases, F. Phytate in foods and significance for humans: Food sources, intake, processing, bioavailability, protective role and analysis. Mol. Nutr. Food Res. 2009, 53, S330-S375, doi:10.1002/mnfr.200900099.

69. FAO; World Health Organization Vitamin and mineral requirements in human nutrition Second edition. World Heal. Organ. 2004, doi:92 41546123.

70. Ismail, H.; Dragišic Maksimovic, J.; Maksimovic, V.; Shabala, L.; Branka, D.Ž.; Tian, Y.; Jacobsen, S.; Shabala, S. Rutin , a fl avonoid with antioxidant activity, improves plant salinity tolerance by regulating $\mathrm{K}+$ retention and $\mathrm{Na}+$ exclusion from leaf mesophyll in quinoa and broad beans. Funct. P 2016, 43, 75-86, doi:doi.org/10.1071/FP15312.

71. Nsimba, R.Y.; Kikuzaki, H.; Konishi, Y. Food Chemistry Antioxidant activity of various extracts and fractions of Chenopodium quinoa and Amaranthus spp . seeds. 2008, 106, 760-766, doi:10.1016/j.foodchem.2007.06.004. 
72. Girgih, A.T. Structural and functional characterization of hemp seed (Cannabis sativa L.) protein-derived antioxidant and antihypertensive peptides, University of Manitoba, 2013.

73. Abderrahim, F.; Huanatico, E.; Segura, R.; Arribas, S.; Gonzalez, M.C.; Condezo-Hoyos, L. Physical features, phenolic compounds, betalains and total antioxidant capacity of coloured quinoa seeds (Chenopodium quinoa Willd.) from Peruvian Altiplano. Food Chem. 2015, 183, 83-90, doi:10.1016/j.foodchem.2015.03.029.

74. Miranda, M.; Vega-gálvez, A.; Martínez, E. a; López, J.; Marín, R.; Aranda, M.; Fuentes, F. Influence of contrasting environments on seed composition of two quinoa genotypes : nutritional and functional properties. Chil. J. Agric. Res. 2013, 73, 108-116, doi:10.4067/S0718-58392013000200004.1.

75. Lozano, M.; Tícona, E.; Carrasco, C.; Flores, Y.; Almanza, G.R. CUANTIFICACIÓN DE SAPONINAS EN RESIDUOS DE QUINUA REAL CHENOPODIUM QUINOA WILLD. Rev. Boliv. Química 2012, 29, 131-138.

76. AOAC INTERNATIONAL Annual Meeting \& Exposition - AOAC INTERNATIONAL.

77. Nascimento, A.C.; Mota, C.; Coelho, I.; Gueifão, S.; Santos, M.; Matos, A.S.; Gimenez, A.; Lobo, M.; Samman, N.; Castanheira, I. Characterisation of nutrient profile of quinoa (Chenopodium quinoa), amaranth (Amaranthus caudatus), and purple corn (Zea mays L.) consumed in the North of Argentina: Proximates, minerals and trace elements. Food Chem. 2014, 148, 420-426, doi:10.1016/j.foodchem.2013.09.155.

78. Benzie, I.F.F.; Strain, J.J. The ferric reducing ability of plasma (FRAP) as a measure of "antioxidant power": The FRAP assay. Anal. Biochem. 1996, 239, 70-76, doi:10.1006/abio.1996.0292.

79. Ainsworth, E.A.; Gillespie, K.M. Estimation of total phenolic content and other oxidation substrates in plant tissues using Folin-Ciocalteu reagent. Nat. Protoc. 2007, 2, 875-877, doi:10.1038/nprot.2007.102.

80. Valenzuela Bustamante, P.D. Evaluación de la actividad antioxidante y determinación del contenido de fenoles totales y flavonoides de hojas de diferentes genotipos de Ugni molinae Turcz. 2015, 65-65.

81. Mohammadi, S.A.; Prasanna, B.M. Analysis of Genetic Diversity in Crop Plants - Salient Statistical Tools and Considerations. Crop Sci. 2003, 43, 1235-1248, doi:10.2135/CROPSCI2003.1235.

82. McGinty, E.M.; Murphy, K.M.; Hauvermale, A.L. Seed dormancy and preharvest sprouting in quinoa (Chenopodium quinoa willd.). Plants 2021, 10, 1-14, doi:10.3390/PLANTS10030458.

83. Martínez, E.A. Quinoa: nutritional aspects of the rice of the Incas. In State of the Art Report of Quinoa in the World in 2013.; Bazile, D., Bertero, H.D., Nieto, C., Eds.; 2015; pp. 278-285.

84. Ruales, J.; Nair, B.M. Saponins, phytic acid, tannins and protease inhibitors in quinoa (Chenopodium quinoa, Willd) seeds. Food Chem. 1993, 48, 137-143, doi:10.1016/0308-8146(93)90048-K.

85. Quiroga, C. Traditional processes and technological innovations in quinoa harvesting, processing, and industrialization. In State of the Art Report of Quinoa in the World in 2013.; Bazile, D., Bertero, H.D., Nieto, C., Eds.; 2015; pp. $218-249$.

86. Jacobsen, S.; Bendevis, M.A. The scope for adaptation of quinoa in Northern Latitudes of Europe. In State of the Art Report of Quinoa in the World in 2013.; 2015; pp. 603-613.

87. Pulvento, C.; Riccardi, M.; Lavini, A.; Iafelice, G.; Marconi, E.; D'Andria, R. Yield and Quality Characteristics of Quinoa Grown in Open Field Under Different Saline and Non-Saline Irrigation Regimes. J. Agron. Crop Sci. 2012, 198, 254-263, doi:10.1111/j.1439-037X.2012.00509.x. 